\title{
Heat and Mass Transfer in a Viscous Nanofluid Containing a Gyrotactic Micro-Organism over a Stretching Cylinder
}

\author{
Mohammad Ferdows ${ }^{1,2,3, *}$, M. Gnaneswara Reddy ${ }^{4}\left(\mathbb{D}\right.$, Faris Alzahrani ${ }^{1}$ and Shuyu Sun ${ }^{3}(\mathbb{D}$ \\ 1 Department of Mathematics, King Abdulaziz University, Jeddah 21589, Kingdom of Saudi Arabia; \\ falzahrani1@kau.edu.sa \\ 2 Research Group of Fluid Flow Modeling and Simulation, Department of Applied Mathematics, \\ University of Dhaka, Dhaka 1000, Bangladesh \\ 3 Applied Mathematics and Computational Science Division, King Abdullah University of Science and \\ Technology, Thuwal 23955-6900, Kingdom of Saudi Arabia; shuyu.sun@kaust.edu.sa \\ 4 Department of Mathematics, Acharya Nagarjuna University Campus, Ongole 523 001, India; \\ mgrmaths@gmail.com \\ * Correspondence: ferdows@du.ac.bd
}

Received: 24 July 2019; Accepted: 3 September 2019; Published: 5 September 2019

\begin{abstract}
This work consists of a theoretical boundary layer analysis of heat and mass transport in a viscous fluid-embracing gyrotactic micro-organism over a cylinder. The flow governing equations are modeled through boundary layer approximations. The governing non-linear partial differential equations are lessened to a set of nonlinear ordinary differential equations using similitude transformation. The boundary layer equations are elucidated numerically, applying the spectral relaxation method with the aid of the computational software MATLAB. The impact of several pertinent parameters on flow convective characteristic phenomena are explored through the use of graphs and tables and are discussed with in-depth physical descriptions. In addition, the friction factor, the rate of heat transfer, rate of mass transfer, and the density number of the motile microorganism are also presented with respect to the above controlled parameters. It is noticed that for the increasing values of the magnetic parameter with velocity reductions and temperature enhancements, the density of the motile microorganism is a declining function of Peclet, Bioconvection Lewis and Bioconvection parameter and the concentration field enhances with the strengthening of Curvature, Magnetic and Thermophoresis, whereas it reduces with the rise of Brownian motion and Lewis parameters. Furthermore, the streamline patters are emphasized for the impact of controlled flow variables. Current outcomes are compared with the available results from previous cases and are observed to be in agreement.
\end{abstract}

Keywords: boundary layer flow; gyrotactic micro-organism; stretching cylinder; magnetic effect; heat and mass transfer; spectral relaxation method

\section{Introduction}

The motion of heat for a viscous fluid has numerous industrialized, biomedical, and engineering demands, such as in capacity generators, petroleum production, plasma research, cancer therapy, the laminar boundary layer dominance in aerodynamics, and numerous others. But the concept has taken a long journey to attain significance. In 1904, Prandtl suggested the laminar boundary layer theory, which suggests that the viscous impact would be limited to thin shear surfaces adjacent borders in the case of flow of fluids with very small viscosity [1]. Sakiadis [2,3] has studied boundary layer Blasius motion from a surface applied with constant speed from a slit into a liquid at rest. Crane [4] 
has investigated the flow over a stretching sheet. Numerous researchers, for instance Dutta et al. [5], Char [6], Gupta, and Gupta [7] expanded the research of Crane [4]. Various features of such problems have been studied by different researchers, for instance Hayat et al. [8], Hayat et al. [9], Xu and Liao [10], and Cortell [11,12]. Two dimensional boundary layer flow across a stretching cylinder was invoked by Datta et al. [13]. Elabarbary [14] analyzed the heat transfer effects on micropolar across a stretching cylinder. Wang [15] has investigated the steady two dimensional flow of a fluid across a stretching cylinder. Ishak et al. [16] have studied the magnetohydrodynamic (MHD) motion and transfer of heat past a stretching cylinder. Ishak et al. [17] have carried out numerical investigations on the effects of suction/blowing on the motion across a stretching cylinder. Butt et al. [18] have explored heat transfer effects on hydromagnetic entropy generation flow over a horizontal stretching cylinder. They obtained solutions by using the bvp4c technique in MATLAB.

In current years, immense efforts have been made regarding the study of nanofluids. The idea behind its popularity is very simple. As we know, regular heat transfer fluids like reservoir, antifreeze, and grease have slight heat transfer abilities due to their short thermal conductivity, whereas alloys have superior thermal conductivities compared to these liquids. Thus, scattering elevated thermal conductive solid particles in a standard heat transfer fluid may raise the thermal conductivity of the fluid. The term "nanofluid" was first suggested by Choi [19]. Planed colloids consists of nanoparticles scattered in a foundation fluid. The foundation fluid is normally a conductive fluid, e.g., water or ethylene glycol. Other foundation fluids include bio-fluids, polymer solutions, oils, and various lubricants. One of the outstanding characteristics of nanofluids are their thermal conductivity [20]. The nanoparticles used in combination with nanofluids are usually $\mathrm{Al}, \mathrm{Cu}, \mathrm{Al}_{2} \mathrm{O}_{3}, \mathrm{TiO}_{2}, \mathrm{AlN}, \mathrm{SiN}, \mathrm{SiC}$, or are carbon nanotubes with a thickness that ranges from $10 \mathrm{~nm}$ to $100 \mathrm{~nm}$.

Recently, nanofluids comprising gyrotactic microorganisms have caused many authors to investigate their various effects on fluid flow problems. Microorganisms play a very important role in reducing the greenhouse effect. Microorganisms, such as dictums, foam, and germs, are more efficient at riveting $\mathrm{Co}_{2}$ compared to trees. Therefore, for the prognosis of the conservatory impact, analyzing the microbes' ecology and putting a precise value on their contribution is crucial. Platt [21] used the term bioconvection, which is a subdivision of biological fluid mechanics. Kuznetsov [22] has investigated the theory of bio-thermal convection with two different microorganisms; gyrotactic and oxytactic. They obtained the solutions using the galerkin method. Nield et al. [23] have explored the combined bio-convection problem over a horizontal layer. They obtained the solutions using the collocation method. Kuznetsov [24] has studied thermal bioconvection of an oxytactic porous medium. They obtained the solutions using the galerkin method. Avraemnko [25] has studied gyrotactic microorganisms over a porous layer. Alloui et al. [26] have studied heat transfer effects on bioconvection suspension of gyrotactic microorganisms. Mahdy [27] has explored mixed convection nanofluid flow across an isothermal wedge. Tham et al. [28] have analyzed steady two dimensional mixed convection flow across a circular cylinder. Kuznetsov [29] has scrutinized bioconvective suspension containing both nanoparticles and gyrotactic microorganisms. Many surveys have been made by many researchers in several models [30-32].

The above literature discloses that nanofluids consist of gyrotactic microorganisms embedded in a cylinder, and demonstrate the combined effects of magnetic field, nanoparticles, and microorganisms across a stretching cylinder. By using similarity transformations, partial differential equations can be transformed into ordinary differential equations and explained numerically by applying the spectral relaxation method (SRM). Outcomes are represented through graphs for the parameters concerned.

\section{Governing Equations}

We examine the axi-symmetric, steady, two dimensional laminar boundary layer incompressible motion of a nanofluid consisting of gyrotactic microorganisms past an extending cylinder. Here, the cylinder is considered to be the $x$-axis and the radius is considered to be the $y$-axis (Figure 1 ). The magnetic field of strength is $B_{0}$. Additionally, it is supposed that the cylinder is extended in the 
axial route, having a velocity of $U_{w}=U_{0}(x / l)$, where $U_{0}$ is constant and $l$ is the characteristic length. The nanofluid has the constant temperature $T_{w}$ and the constant concentration $C_{w}$ along the stretching cylinder. Under these suppositions, the governing equations are [33-37]:

$$
\begin{gathered}
\frac{\partial}{\partial x}(r u)+\frac{\partial}{\partial r}(r v)=0 \\
u \frac{\partial u}{\partial x}+v \frac{\partial u}{\partial r}=v\left(\frac{\partial^{2} u}{\partial r^{2}}+\frac{1}{r} \frac{\partial u}{\partial r}\right)-\frac{\sigma_{e} B_{0}^{2}}{\rho} u \\
u \frac{\partial T}{\partial x}+v \frac{\partial T}{\partial r}=\alpha\left(\frac{\partial^{2} T}{\partial r^{2}}+\frac{1}{r} \frac{\partial T}{\partial r}\right)+\frac{v}{c_{p}}\left(\frac{\partial u}{\partial r}\right)^{2}+\tau\left(D_{B}\left(\frac{\partial T}{\partial r} \frac{\partial C}{\partial r}\right)+\frac{D_{T}}{T_{\infty}}\left(\frac{\partial T}{\partial r}\right)^{2}\right) \\
u \frac{\partial C}{\partial x}+v \frac{\partial C}{\partial r}=D_{B}\left(\frac{\partial^{2} C}{\partial r^{2}}+\frac{1}{r} \frac{\partial C}{\partial r}\right)+\frac{v}{c_{p}}\left(\frac{\partial u}{\partial r}\right)^{2}+\frac{D_{T}}{T_{\infty}}\left(\frac{\partial^{2} T}{\partial r^{2}}\right) \\
u \frac{\partial n}{\partial x}+v \frac{\partial n}{\partial r}=D_{n}\left(\frac{\partial^{2} n}{\partial r^{2}}+\frac{1}{r} \frac{\partial n}{\partial r}\right)+\frac{\partial W_{c}}{C_{w}-C_{\infty}} \frac{\partial}{\partial r}\left(n \frac{\partial C}{\partial r}\right)
\end{gathered}
$$

and the pertinent boundary conditions are:

$$
\begin{gathered}
\text { At } r=a: u=U_{w}, v=0, T=T_{w}, C=C_{w}, n=n_{w} \\
\quad \text { As } r \rightarrow \infty: u \rightarrow 0, T \rightarrow T_{\infty}, C \rightarrow C_{\infty}, n \rightarrow n_{\infty}
\end{gathered}
$$

where $x$ and $r$ are coordinates steady in the axial and radial route of the cylinder, respectively, $u$ and $v$ are the velocity parts along the $x$ and $r$ directions, $T$ indicates the temperature, $D_{B}$ is the Brownian diffusion coefficient, $D_{T}$ is the thermophoresis coefficient, $B_{0}$ is the magnetic field strength, $\sigma_{\mathcal{e}}$ is the electrical conductivity, $n$ is the density of the motile microorganism, $c_{p}$ is the specific heat at constant pressure, $C_{w}$ is the concentration at the surface, $C_{\infty}$ is the concentration in the free stream, $D_{n}$ is the diffusivity of the microorganisms, $\tau$ is the effective heat capacitance, $W_{c}$ is the constant maximum cell swimming speed, $\alpha$ is the thermal diffusivity, $v$ is the kinematic viscosity, and $a$ is the radius of the cylinder.

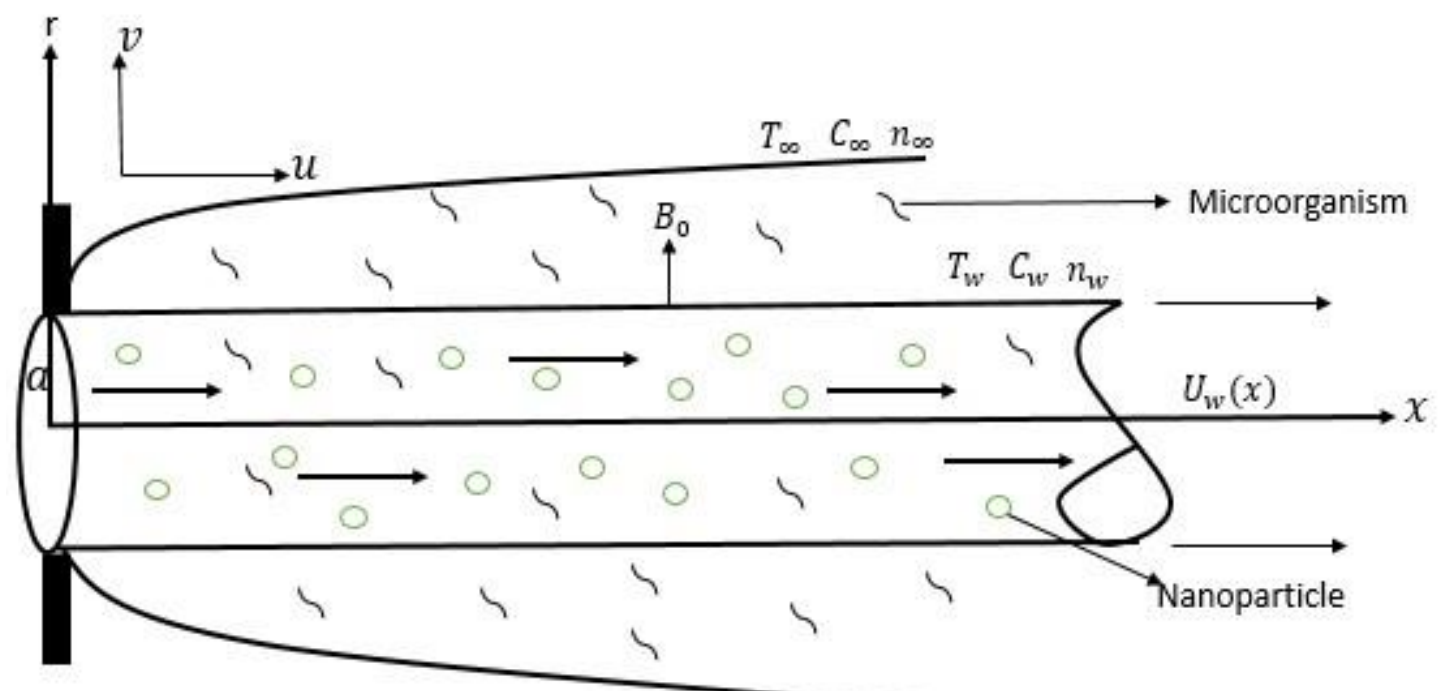

Figure 1. Physical diagram and co-ordinate structure. 
Now, using the following similarity transformations [33,36]:

$$
\begin{gathered}
\eta=\frac{r^{2}-a^{2}}{2 a}\left(\frac{U_{w}}{v x}\right)^{\frac{1}{2}}, \psi=\left(v U_{w} x\right)^{\frac{1}{2}} a f(\eta), \phi(\eta)=\frac{C-C_{\infty}}{C_{w}-C_{\infty}} \\
\chi(\eta)=\frac{n-n_{\infty}}{n_{w}-n_{\infty}}, \theta(\eta)=\frac{T-T_{\infty}}{T_{w}-T_{\infty}}
\end{gathered}
$$

the transformed equations are

$$
\begin{gathered}
(1+2 \gamma \eta) f^{\prime \prime \prime}+(2 \gamma+f) f^{\prime \prime}-M f^{\prime}-f^{\prime 2}=0 \\
(1+2 \gamma \eta) \theta^{\prime \prime}+(2 \gamma+\operatorname{Pr}) f \theta \prime+(1+2 \gamma \eta) \operatorname{Pr} N b \theta \prime \phi \prime \\
+\operatorname{Pr} E c(1+2 \gamma \eta) f \prime^{\prime 2}+\operatorname{Pr} N t(1+2 \gamma \eta) \theta \prime^{\prime 2}=0 \\
(1+2 \gamma \eta) \varphi^{\prime \prime}+2 \gamma \varphi^{\prime}+\left(\frac{N t}{N b}\right)\left(\gamma \theta^{\prime}+(1+2 \gamma \eta) \theta^{\prime \prime}\right)+L e f \varphi^{\prime}=0 \\
(1+2 \gamma \eta) \chi^{\prime \prime}+2 \gamma \chi^{\prime}+\operatorname{Lb} \operatorname{Pr} f \chi^{\prime}-\operatorname{Pe}\left(\begin{array}{c}
(1+2 \gamma \eta) \varphi^{\prime} \chi^{\prime}+\gamma \chi \varphi^{\prime} \\
+(1+2 \gamma \eta) \chi \varphi^{\prime \prime}+\sigma \gamma \varphi^{\prime} \\
+\sigma(1+2 \gamma \eta) \varphi^{\prime \prime}
\end{array}\right)=0
\end{gathered}
$$

and the refreshed boundary conditions are

$$
\begin{gathered}
\eta \rightarrow 0: \quad f^{\prime}(0)=1, \theta(0)=1, \phi(0)=1, \chi(0)=1 \\
\eta \rightarrow \infty: \quad f^{\prime}(\infty)=0, \theta(\infty)=0, \phi(\infty)=0, \chi(\infty)=0
\end{gathered}
$$

Here, the magnetic field parameter $M=\left(\frac{\sigma B_{0}^{2} l}{\rho U_{0}}\right)$, the curvature parameter $\gamma=\left(\frac{v l}{U_{0} a^{2}}\right)^{\frac{1}{2}}$, the Prandtl number $\operatorname{Pr}=\frac{v}{\alpha}$, the Peclet number $P e=\left(\frac{d w c}{D_{n}}\right)$, the Brownian motion parameter $N b=\left(\frac{\left(\rho c_{p}\right) D_{B}\left(C_{w}-C_{\infty}\right)}{v T_{\infty}\left(\rho c_{f}\right)}\right)$, the thermophoresis parameter $N t=\left(\frac{\left(\rho c_{p}\right) D_{T}\left(T_{w}-T_{\infty}\right)}{v T_{\infty}\left(\rho c_{f}\right)}\right)$, the Lewis number $L e=\frac{v}{D_{B}}$, the bioconvection Lewis number $L b=\frac{\alpha}{D_{n}}$, the bioconvection parameter $\sigma=\frac{n_{\infty}}{n_{w}-n_{\infty}}$, and the Eckert number $E c=\left(\frac{U_{w}^{2}}{c_{p}\left(T_{w}-T_{\infty}\right)}\right)$.

The physical quantities of note are the skin friction coefficient, $C_{f}$, the local Nusselt number, $N u_{x}$, the local Sherwood number, $S h_{x}$, and the density number of the motile microorganism, $N n_{x}$ which are given by

$$
C_{f}=\frac{2 \tau_{w}}{\rho U_{w}^{2}}, S h_{x}=\frac{x q_{w}}{k \Delta T}, N u_{x}=\frac{x q_{m}}{D_{B} \Delta C}, N n_{x}=\frac{x q_{n}}{D_{n} \Delta n}
$$

where $\tau_{w}=-\mu(\partial u / \partial r)_{r=a}$ is the surface shear stress, $q_{w}=-k(\partial T / \partial r)_{r=a}$ is the surface heat flux, $q_{m}=-D_{B}(\partial C / \partial r)_{r=a}$ is the surface mass flux, and $q_{n}=-D_{n}(\partial n / \partial r)_{r=a}$ is the surface motile microorganism flux.

Substituting the usual similarity transformations into Equation (15) yields:

$$
\frac{1}{2} C_{f} \sqrt{\operatorname{Re}} \bar{x}=-f^{\prime \prime}(0), \frac{N u_{x}}{\sqrt{\operatorname{Re}}} \bar{x}=-\theta^{\prime}(0), \frac{S h_{x}}{\sqrt{\operatorname{Re}}} \bar{x}=-\phi^{\prime}(0), \frac{N n_{x}}{\sqrt{\operatorname{Re}}} \bar{x}=-\chi^{\prime}(0)
$$

where $\operatorname{Re}=U_{w} l / v, \bar{x}=\sqrt{\frac{x}{l}}$.

\section{Spectral Relaxation Method and Comparison}

The spectral relaxation method is a numerical technique established using a simple iteration scheme established by reducing large structures of nonlinear equations into small structures of linear equations [37]. The technique has been useful in several science and engineering problems described 
by coupled nonlinear systems of ordinary or partial differential equations. The basic idea of the spectral relaxation method approach is the easy decoupling and rearrangement of the nonlinear differential equations in a Gauss-Seidel approach to obtain a linear system of equations. The resulting spectral relaxation method iterative scheme is then integrated, employing the collocation method to obtain the numerical solution. The technique was introduced by Motsa and Makukula [38] for the investigation of the von Karman motion of a Reiner-Rivlin liquid under the influence of ohmic heating and viscous dissipation. The spectral relaxation method has been found to be accurate and rapidly convergent to the numerical results. Shateyi [39] has used the spectral relaxation method to calculate the hydromagnetic motion and heat transfer in a Maxwell liquid past a stretching sheet under the influence of chemical reaction and thermophoresis in a permeable medium. Shateyi and Makinde [40] have studied the heat transfer effects on hydromagnetic flow across a cylinder. Magneto hydrodynamic two dimensional non-Newtonian fluid across a stretching sheet has been analyzed by Shateyi and Marewo [41]. Motsa et al. [42] have proposed a new multi-stage numerical method that was applied to the numerical result of 3-hyperchaotic structures. Motsa et al. [43] have proposed and applied a new pseudo-spectral technique for explaining the nonlinear initial value problems in the presence of chaotic properties. While Nik and Rebelo [44] were solving the hyper-chaotic complex systems, the exactness and reliability of the spectral relaxation method against the MATLAB bvp4c technique. Motsa et al. [45] have extended the application of the spectral relaxation method to systems of nonlinear partial differential equations that model unsteady boundary layer motion. The exactness of the spectral relaxation method in solving nonlinear PDEs was determined by equating the computational performance of the spectral relaxation method against the spectral quasi-linearization method and Keller-box method. Awad et al. [46] have studied unsteady motion past an extending sheet in a viscous fluid with chemical reaction and have solved the boundary layer equations by the spectral relaxation method. Recently, Haron et al. [47] have applied the spectral relaxation method to the unsteady magneto hydrodynamic combined convective motion of a nanofluid past a stretching/shrinking surface in the presence of a heat source, internal fraction, thermal diffusion, and diffusion thermo effects. In this work we will use the SRM to solve systems of highly nonlinear ordinary differential equations that describe some fluids' motion, transfer of heat, and mass. The spectral relaxation method is considered to be exact and promptly convergent to the numerical outcomes.

For our model, we discretize the transformed Equation (9) to Equation (14) using the following spectral relaxation method algorithm:

1. By using $f^{\prime}(\eta)=F(\eta)$ the velocity equation can transformed in terms of $F(\eta)$.

2. Supposing that $f(\eta), \theta(\eta), \phi(\eta), \chi(\eta)$ are familiar since past iterations (symbolized by $f_{r}(\eta), \theta_{r}(\eta), \phi_{r}(\eta), \chi_{r}(\eta)$, ) form an iteration scheme for $F(\eta), \theta(\eta), \phi(\eta), \chi(\eta)$ by supposing that only linear terms in $F(\eta) \theta(\eta), \phi(\eta), \chi(\eta)$ are to be calculated at the present iteration level (indicated by $F_{r+1}(\eta), \theta_{r+1}(\eta), \phi_{r+1}(\eta), \chi_{r+1}(\eta)$ ) and all dissimilar expressions are supposed to be familiar from the past iteration. Further, nonlinear terms in $F(\eta), \theta(\eta), \phi(\eta), \chi(\eta)$ are calculated at the past iteration.

In the text of the SRM reported above, Equations (9)-(14) are transformed into

$$
(1+2 \gamma \eta) F_{r+1}^{\prime \prime}+\left(2 \gamma+f_{r}\right) F_{r+1}^{\prime}-M F_{r+1}-F_{r}^{2}=0
$$

$(1+2 \gamma \eta) \theta^{\prime \prime}{ }_{r+1}+(2 \gamma+\operatorname{Pr}) f_{r} \theta_{r+1}^{\prime}+(1+2 \gamma \eta) \operatorname{Pr} N b \theta^{\prime}{ }_{r+1} \varphi^{\prime}{ }_{r}+E c \operatorname{Pr}(1+2 \gamma \eta) F^{\prime}{ }_{r}^{2}+$ $\operatorname{PrN} t(1+2 \gamma \eta) \theta^{\prime}{ }^{2}=0$

$$
\begin{aligned}
& (1+2 \gamma \eta) \varphi^{\prime \prime}{ }_{r+1}+2 \gamma \varphi_{r+1}^{\prime}+\left(\frac{N t}{N b}\right)\left(\gamma \theta_{r+1}^{\prime}+(1+2 \gamma \eta) \theta_{r+1}^{\prime \prime}\right)+L e f_{r+1} \varphi_{r+1}^{\prime}=0 \\
& (1+2 \gamma \eta) \chi^{\prime \prime}+2 \gamma \chi^{\prime}+L b \operatorname{Pr} f \chi^{\prime}-\operatorname{Pe}\left((1+2 \gamma \eta) \phi^{\prime} \chi^{\prime}+\gamma \chi \phi^{\prime}+(1+2 \gamma \eta) \chi \phi^{\prime \prime}+\sigma \gamma \phi^{\prime}\right)+ \\
& \operatorname{Pe\sigma }(1+2 \gamma \eta) \phi^{\prime \prime}=0
\end{aligned}
$$


The corresponding boundary conditions are written as:

$$
\begin{array}{ll}
F_{r+1}(0)=1 ; & F_{r+1}(\infty)=0 \\
\theta_{r+1}(0)=1 ; & \theta_{r+1}(\infty)=0 \\
\phi_{r+1}(0)=1 ; & \phi_{r+1}(\infty)=0 \\
\chi_{r+1}(0)=1 ; & \chi_{r+1}(\infty)=0
\end{array}
$$

We implement the collocation technique to explain the decoupled system (17)-(24). In implementing the spectral method, we obtain the unknown function at the $\mathrm{N}+1$ collocation points by requiring that (17)-(20) be fulfilled precisely at all these points. An appropriate set of collocation points are the Gauss-Lobatto points defined $[-1,1]$ by

$$
\tau_{j}=\cos \left(\frac{\pi j}{N}\right), \quad j=0,1,2, \ldots, N
$$

We estimate the derivative of the unrevealed functions, say $f^{\prime}(\eta)$, using the so-called differentiation matrix $D$ of size $(N+1) \times(N+1)$, which is computed as the collocation points of the matrix product vector:

$$
\frac{d f_{r}}{d \eta}=\sum_{k=0}^{N} D_{l k} f_{r}\left(\tau_{k}\right)=D f_{r,} \quad l=0,1,2,3, \ldots, N
$$

where $N+1$ represents the number of collocation grid points, $D=\frac{2 D}{L}$ and the set

$$
f=\left[f\left(\tau_{0}\right), f\left(\tau_{1}\right), f\left(\tau_{2}\right), f\left(\tau_{3}\right), \ldots, f\left(\tau_{N}\right)\right]^{T}
$$

stands for the vector function at the collocation points. The higher-order derivatives are gained as power of $D$,

$$
f_{r}^{(p)}=D^{p} f_{r}
$$

where $p$ stands for the order of the derivative. The variable $\tau$ is defined from the linear transformation $\eta=L \frac{\tau+1}{2}$ to map the interval $[0, L]$ to $[-1,1]$, where $L$ is taken to be large, sufficient to numerically approximate the conditions at infinity. Applying the Chebychev spectral collocation method on (17)-(20), we have

$$
\begin{aligned}
& A_{1} F_{r+1}=B_{1} \\
& A_{2} F_{r+1}=B_{2} \\
& A_{3} \theta_{r+1}=B_{3} \\
& A_{4} \phi_{r+1}=B_{4} \\
& A_{5} \chi_{r+1}=B_{5}
\end{aligned}
$$

where:

$$
\begin{gathered}
A_{1}=\operatorname{diag}[(1+2 \gamma \eta)] D^{2}+\operatorname{diag}\left[\left(2 \gamma+f_{r}\right)\right] D-M I \\
B_{1}=F_{r}^{2} \\
A_{2}=D \\
B_{2}=F_{r+1} \\
A_{4}=\operatorname{diag}[(1+2 \gamma \eta)] D^{2}+\operatorname{diag}\left[\left(2 \gamma+\operatorname{Le} f_{r+1}\right)\right] D \\
B_{4}=-\left(\frac{N t}{N b}\right)\left(\gamma \theta_{r+1}^{\prime}+(1+2 \gamma \eta) \theta_{r+1}^{\prime \prime}\right)
\end{gathered}
$$




$$
\begin{gathered}
A_{5}=\operatorname{diag}(1+2 \gamma \eta) D^{2}+\operatorname{diag}\left(2 \gamma+\operatorname{LbPr} f_{r+1}-P e(1+2 \gamma \eta) \phi^{\prime}{ }_{r+1}\right) D-P e\left[(1+2 \gamma \eta) \phi^{\prime \prime}{ }_{r+1}+\gamma \phi_{r+1}\right] \\
B_{5}=\sigma \gamma{\phi^{\prime}}_{r+1}^{\prime}+\sigma(1+2 \gamma \eta) \phi^{\prime \prime}{ }_{r+1}
\end{gathered}
$$

In Equations (31)-(37), I is an identity matrix. The shape of the matrix is $(N+1) \times 1$ and diag[] stands for a diagonal size $(N+1) \times(N+1)$, where $N$ indicates the number of mesh points $f, F, \theta, \phi, \chi$, respectively. The subscript $r$ indicates the iteration number.

The first guesses to that begin the spectral relaxation method scheme for Equations (32)-(39) are taken as functions that fulfil the boundary conditions. The velocity and temperature descriptions for the boundary layer problem examined in this work decay exponentially at $\eta=\infty$. Take the following exponential function as

$$
f_{0}=1-e^{-\eta}, \quad F_{0}(\eta)=e^{-\eta}, \quad \theta(\eta)=e^{-\eta}, \quad \phi(\eta)=e^{-\eta} \quad \chi(\eta)=e^{-\eta}
$$

In our present study, we take the $N=80$ collocation point. These values give accurate results for all the quantities of physical interest. Starting from the initial approximation (41), the SRM scheme is repeatedly solved until the following condition is satisfied,

$$
\max \left(F_{r+1}-F_{\infty}, G_{r+1}-G_{\infty}, \theta_{r+1}-\theta_{\infty}\right) \leq \varepsilon_{r}
$$

where $\varepsilon_{r}$ is a prescribed error tolerance, which in this study is taken to be $10^{-6}$.

The accuracy of the spectral relaxation method is examined by performing different comparisons at various conditions with previously reported works. Here we have compared the value of the Nusselt number with Khan and Pop [34], Wang [35], and Elbashbeshy et al. [48], taking $M=N t=N b=L e=$ $L b=P e=E c=\sigma=\gamma=0$ and varying the value of Pr. The results obtained are in agreement, as displayed in Table 1. From Table 1, we can observe that in both the present study and the previous studies, the rate of heat transfer increases with the rising values of $\operatorname{Pr}$. When $\operatorname{Pr}=0.2$, the rate of heat transfer is 0.16911012 for the present study and $0.1691,0.1697$, and 0.1690886 for the previous studies, and at $\mathrm{Pr}=70$ the rate of heat transfer is 6.46218077 for the present study and 6.4622, 6.4622, and 6.4621996 for the previous studies.

Table 1. Comparison of numerical values for different values of $\operatorname{Pr}$ on the Nusselt number.

\begin{tabular}{ccccc}
\hline Pr & Present Study & Khan and Pop [34] & Wang [35] & Elbashbeshy et al. [39] \\
\hline 0.2 & 0.16911012 & 0.1691 & 0.1697 & 0.1690886 \\
0.7 & 0.45391616 & 0.4539 & 0.4539 & 0.4539161 \\
2 & 0.91135768 & 0.9114 & 0.9114 & 0.9113577 \\
7 & 1.89540305 & 1.8954 & 1.8954 & 1.8954033 \\
20 & 3.35390414 & 3.3539 & 3.3539 & 3.3539042 \\
70 & 6.46218077 & 6.4622 & 6.4622 & 6.4621996 \\
\hline
\end{tabular}

\section{Discussions}

To analysis our study we have transformed our governing Equations (2)-(5) and boundary restrictions (6)-(7) using similarity variables and have got the transformed Equations (9)-(12) and transformed boundary condition (13)-(14). After transforming, we get the curvature parameter $\gamma$, Prandtl number $P r$, magnetic field parameter $M$, Peclet number $P e$, Lewis number $L e$, bioconvection Lewis number $L b$, Brownian motion parameter $N b$, thermophoresis parameter $N t$, Eckert number $E c$ and bioconvection constant $\sigma$. The computations of our study will be carried out for the different values of these parameters. We use the SRM to discretize our transformed Equations (9)-(14) by using MATLAB to get our required data. For computations we have assigned dimensionless parameter as:

$$
\begin{gathered}
M=0,1,3,6, N t=0.1,0.2,0.5,1.2, N b=0.25,0.5,1.2, L e=1,1.5,2,5, L b=0.2,0.5,0.7 \\
P e=0.3,0.5,0.7, E c=0.01,0.1,0.2, \sigma=0.2,0.4,0.6, \operatorname{Pr}=0.71,1,5,7,6.17, \gamma=0.5,1,2
\end{gathered}
$$


Figures $2-5$ describe the momentum, heat, mass, and density of the motile microorganism profile for several values of the curvature parameter $\gamma$. A cross-over is initiated in the $f \prime(\eta)$ and $\chi(\eta)$ profile frontier layer at $\eta \approx 0.5$, as shown in Figures 2 and 5. For the dynamic region, $\eta<0.5$ the enhancement of $\gamma$ leads to a reduction in the velocity and microorganism boundary layers, because of the small enrichment of the frictional forces, by extending the surface shear stress. On the other hand, for $\eta>0.5$ the opposite takes place as the curvature parameter increases. From Figures 3 and 4 it can be seen that temperature description decreases and concentration description increases with the enhancement of $\gamma$. An explanation for this observation is that enhancement of the curvature parameter results in the reduction of the surface area of the cylinder.

Figures 6-9 illustrate the momentum, heat, mass, and density of the motile microorganism profile for different values of $M$. For increased values of the magnetic parameter $M$, temperature, concentration, and the motile microorganism profiles increases, and the opposite behavior is seen in the velocity profile. $M$ grows a resistance force that works conversely to the flow field and enhances the thermal, solutal, and motile microorganism boundary layer thickness.

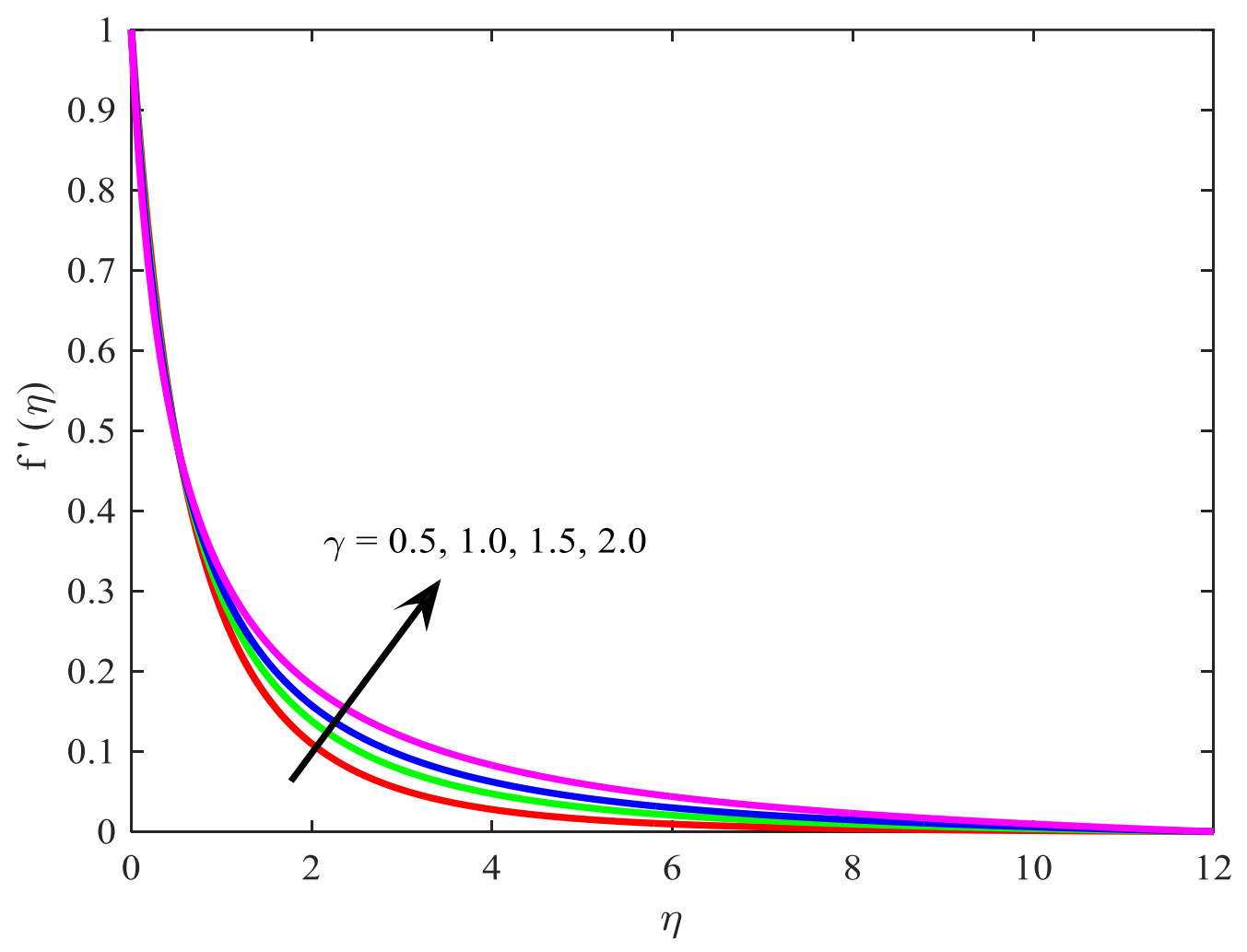

Figure 2. Velocity description, $f^{\prime}(\eta)$ for different values of $\gamma$, where $M=1, N t=0.2, N b=0.5, L e=5$, $L b=0.2, P e=0.3, E c=0.01, \sigma=0.2, \operatorname{Pr}=5$. 


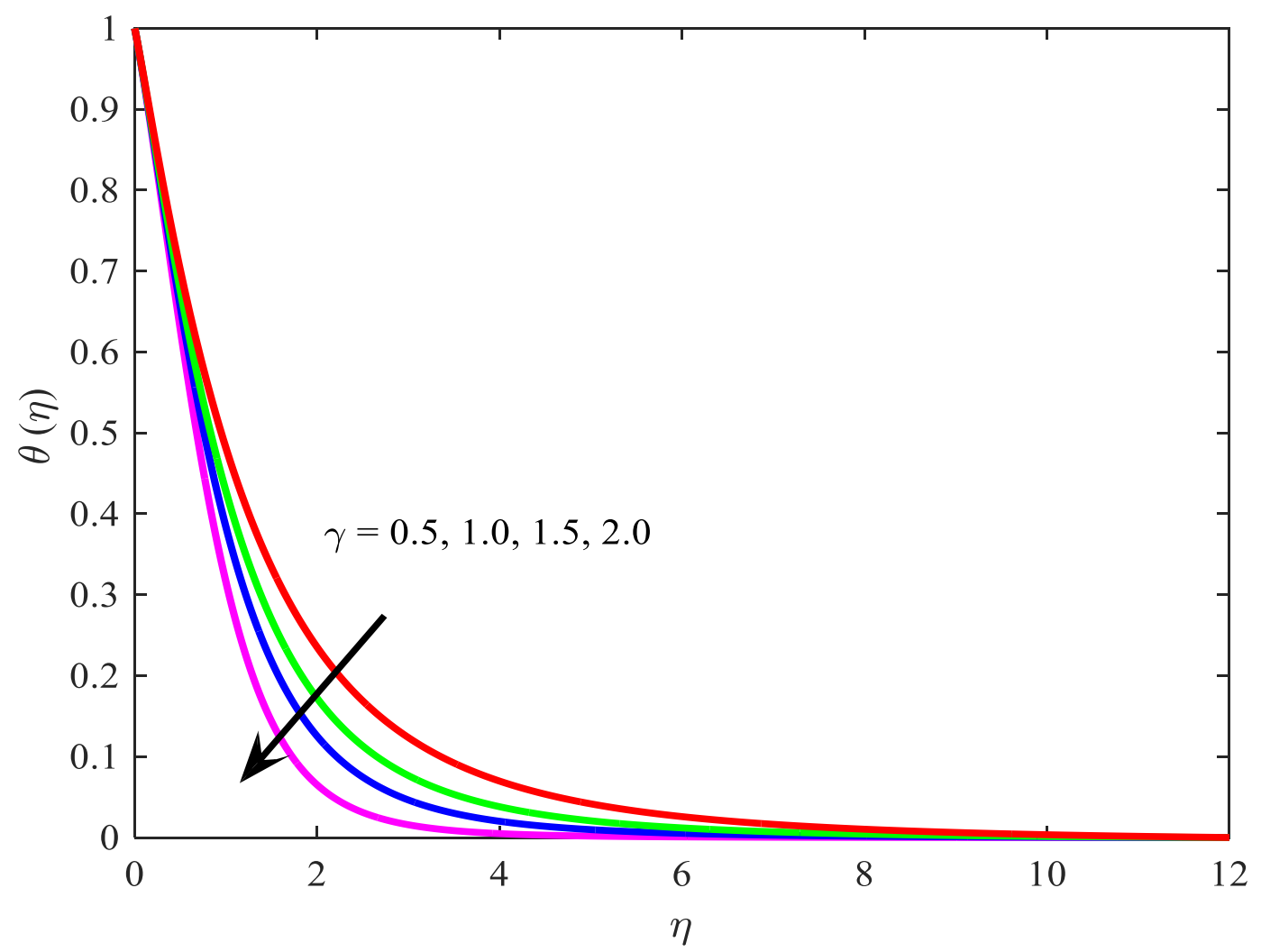

Figure 3. Temperature description, $\theta(\eta)$ for different values of $\gamma$, where $M=1, N t=0.2, N b=0.5$, $L e=0.1, L b=0.2, P e=0.3, E c=0.01, \sigma=0.2, \operatorname{Pr}=5$.

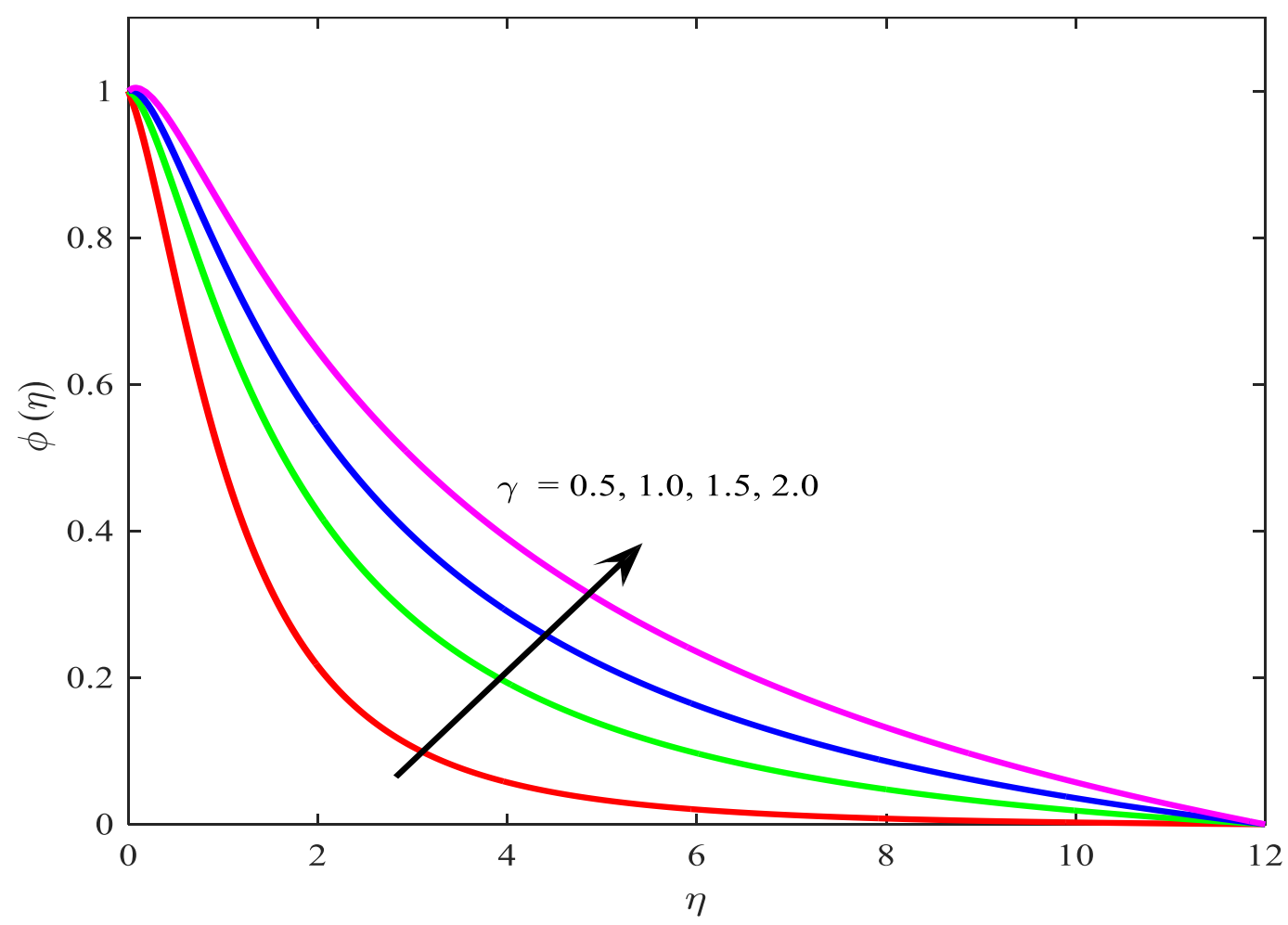

Figure 4. Concentration profiles, $\phi(\eta)$ for various values of $\gamma$, where $M=1, N t=0.2, N b=0.5$, $L e=0.1, L b=0.2, P e=0.3, E c=0.01, \sigma=0.2, \operatorname{Pr}=5$. 


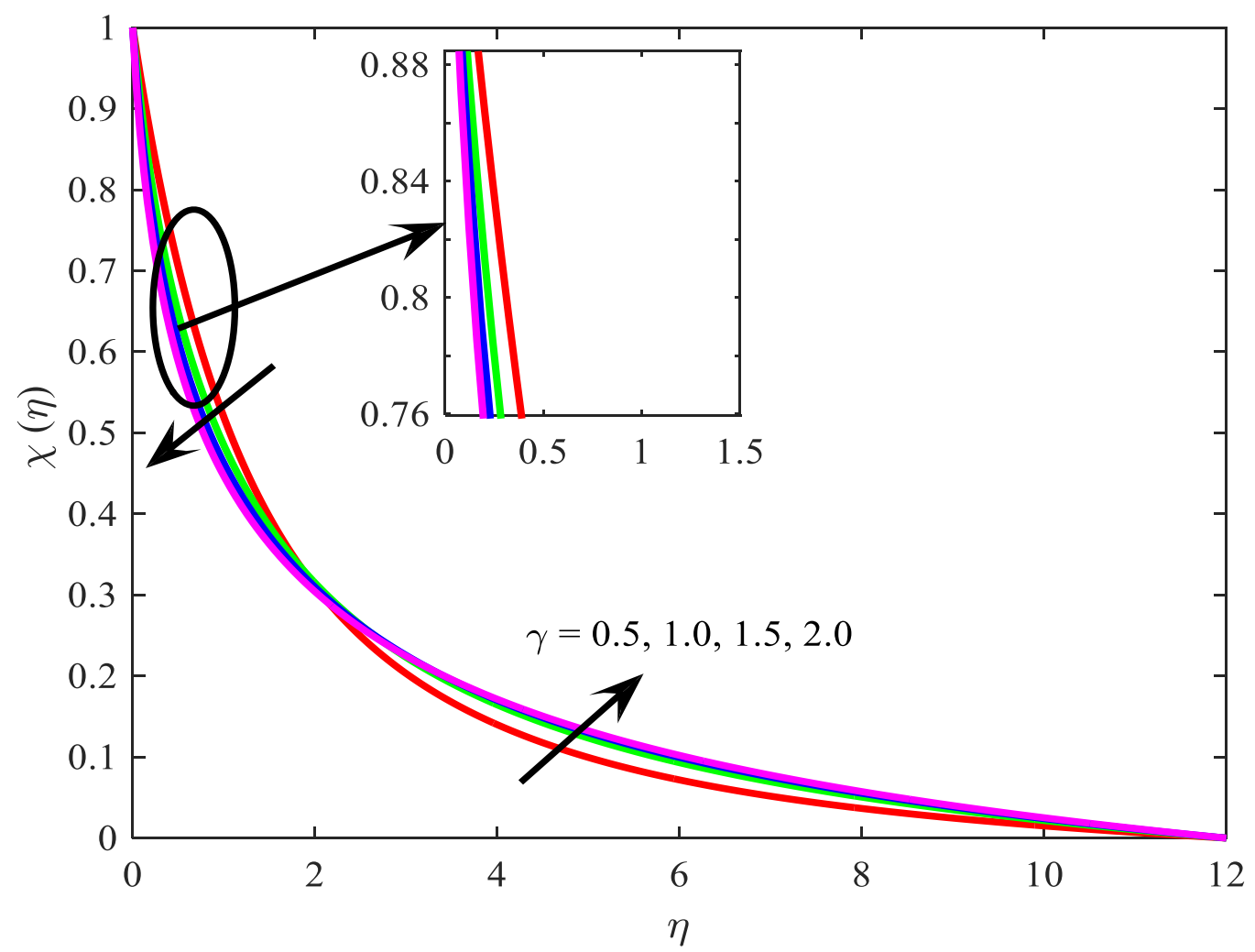

Figure 5. Volume fraction for gyrotactic microorganism profiles, $\chi(\eta)$ for different values of $\gamma$, where $M=1, N t=0.2, N b=0.5, L e=0.1, L b=0.2, P e=0.3, E c=0.01, \sigma=0.2, \operatorname{Pr}=5$.

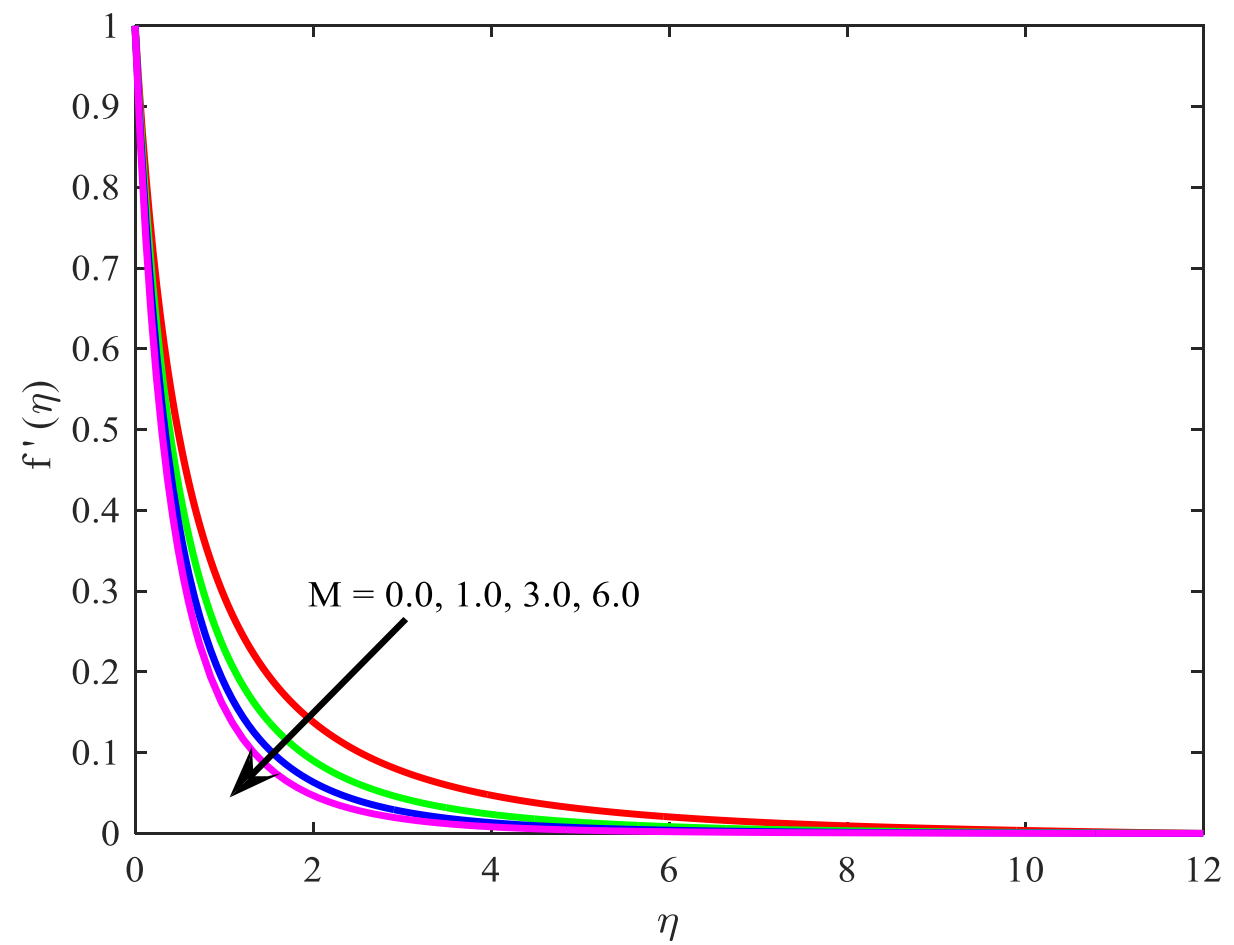

Figure 6. Velocity profiles, $f^{\prime}(\eta)$ for different values of $M$, where $\gamma=1, N t=0.1, N b=0.5, L e=5$, $L b=0.5, P e=0.5, E c=0.2, \sigma=0.2, \operatorname{Pr}=0.71$. 


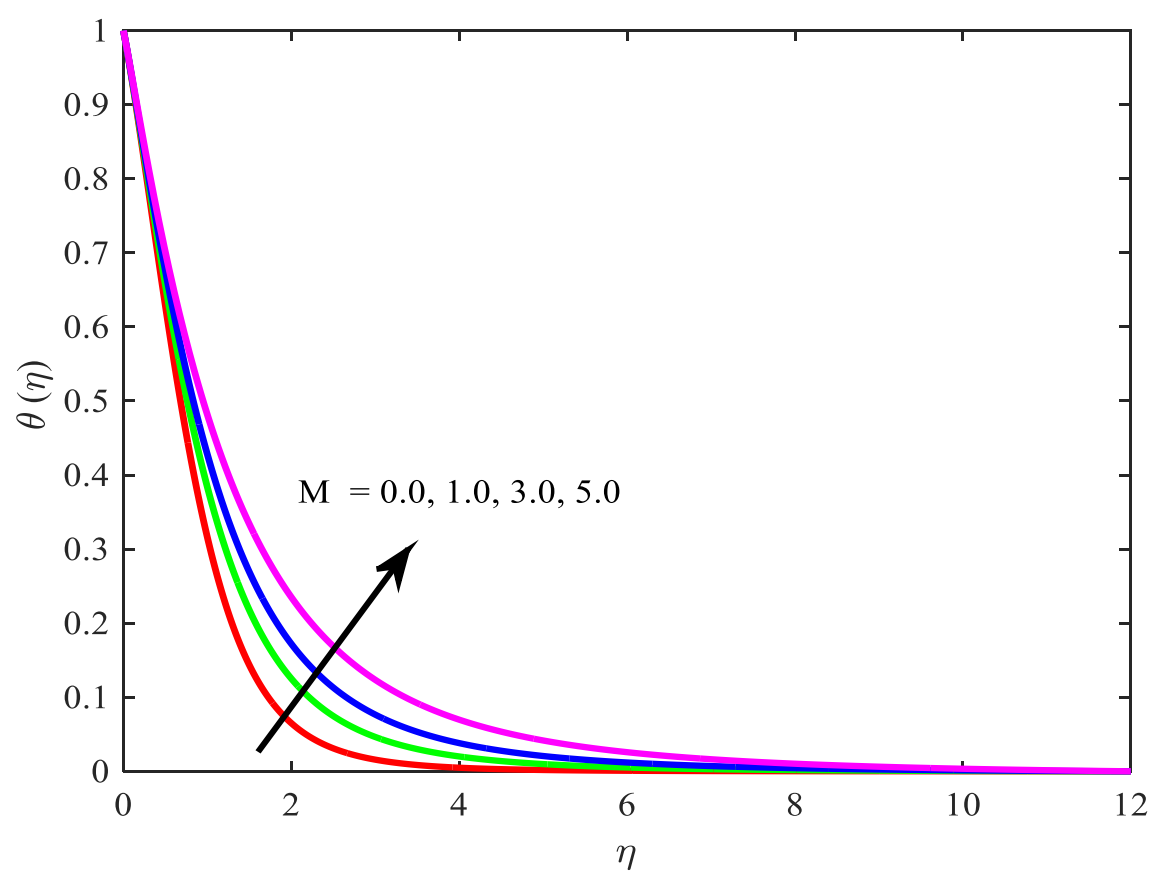

Figure 7. Temperature profiles, $\theta(\eta)$ for different values of $M$, where $\gamma=1, N t=0.1, N b=0.5$, Le $=5$, $L b=0.5, P e=0.5, E c=0.2, \sigma=0.2, \operatorname{Pr}=0.71$.

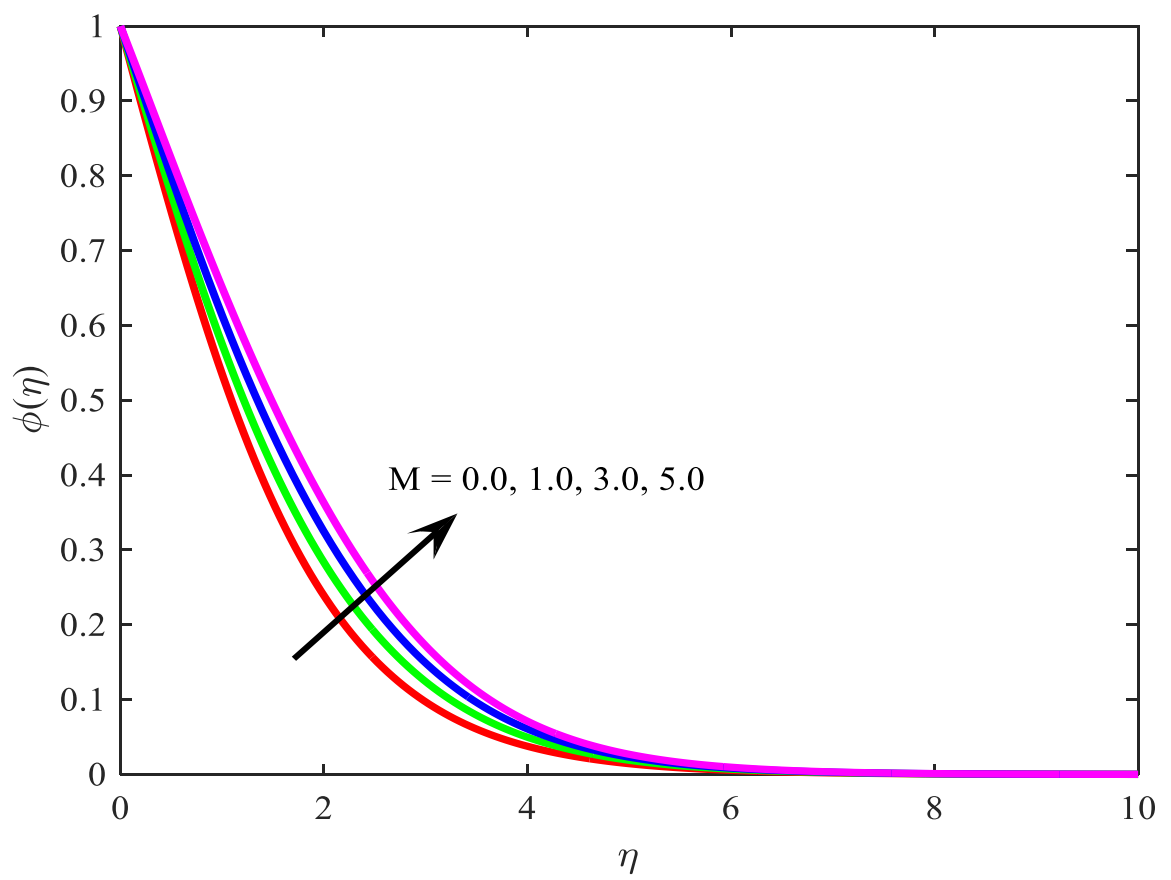

Figure 8. Concentration description, $\phi(\eta)$ for various values of $M$, where $\gamma=1, N t=0.1, N b=0.5$, $L e=5, L b=0.5, P e=0.5, E c=0.2, \sigma=0.2, \operatorname{Pr}=0.71$.

Figures 10-12 describe the $\theta(\eta), \phi(\eta)$ and $\chi(\eta)$ profiles for different values of the Brownian flow parameter $\mathrm{Nb}$. From Figures $10-12$ it is noted that as the value of $\mathrm{Nb}$ enhances, the temperature, concentration, and microorganism description of the nanofluid decreases. Brownian motion is a force generated by the concentration gradient between the cold wall and hot gas effecting the particulate movements towards the cold wall. 


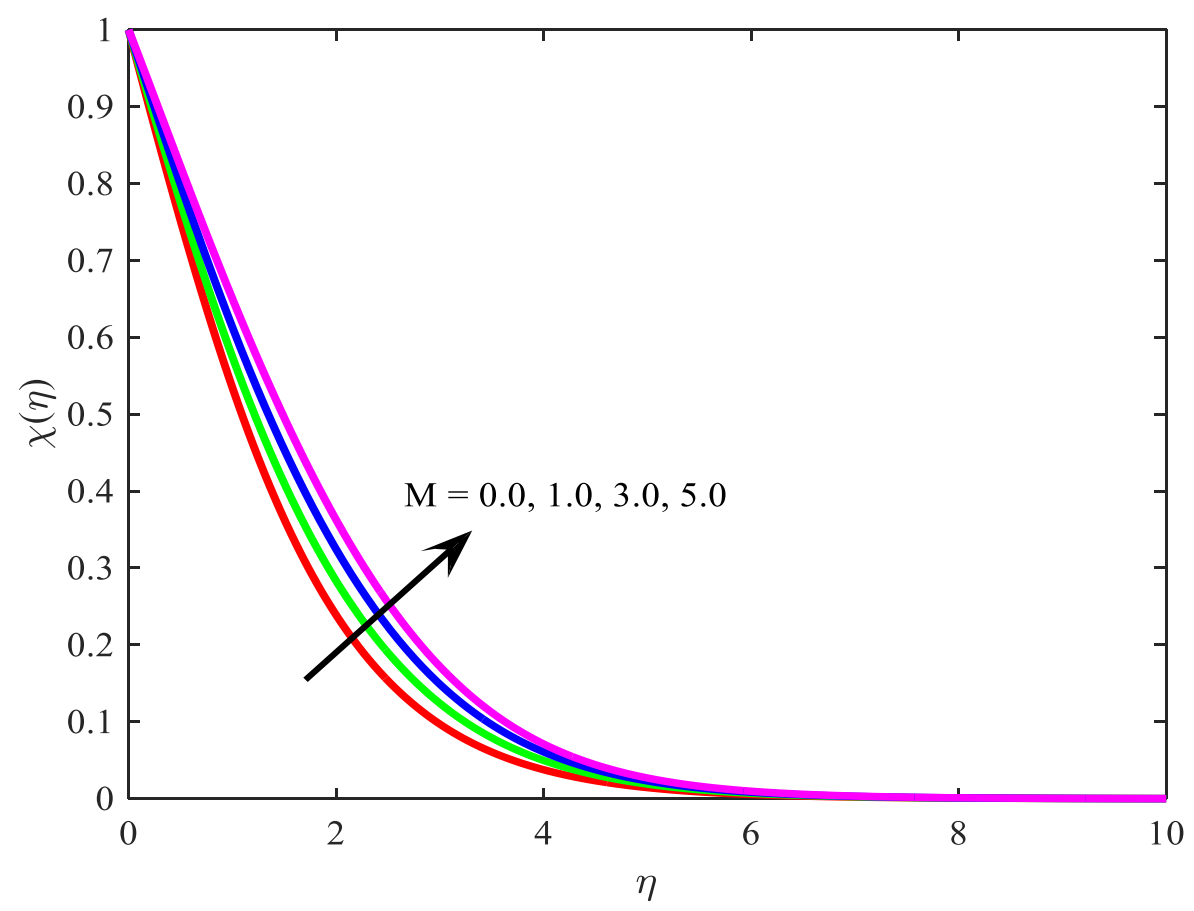

Figure 9. Volume fraction for gyrotactic microorganism profiles, $\chi(\eta)$ for different values of $M$, where $\gamma=1, N t=0.1, N b=0.5, L e=5, L b=0.5, P e=0.5, E c=0.2, \sigma=0.2, \operatorname{Pr}=0.71$.

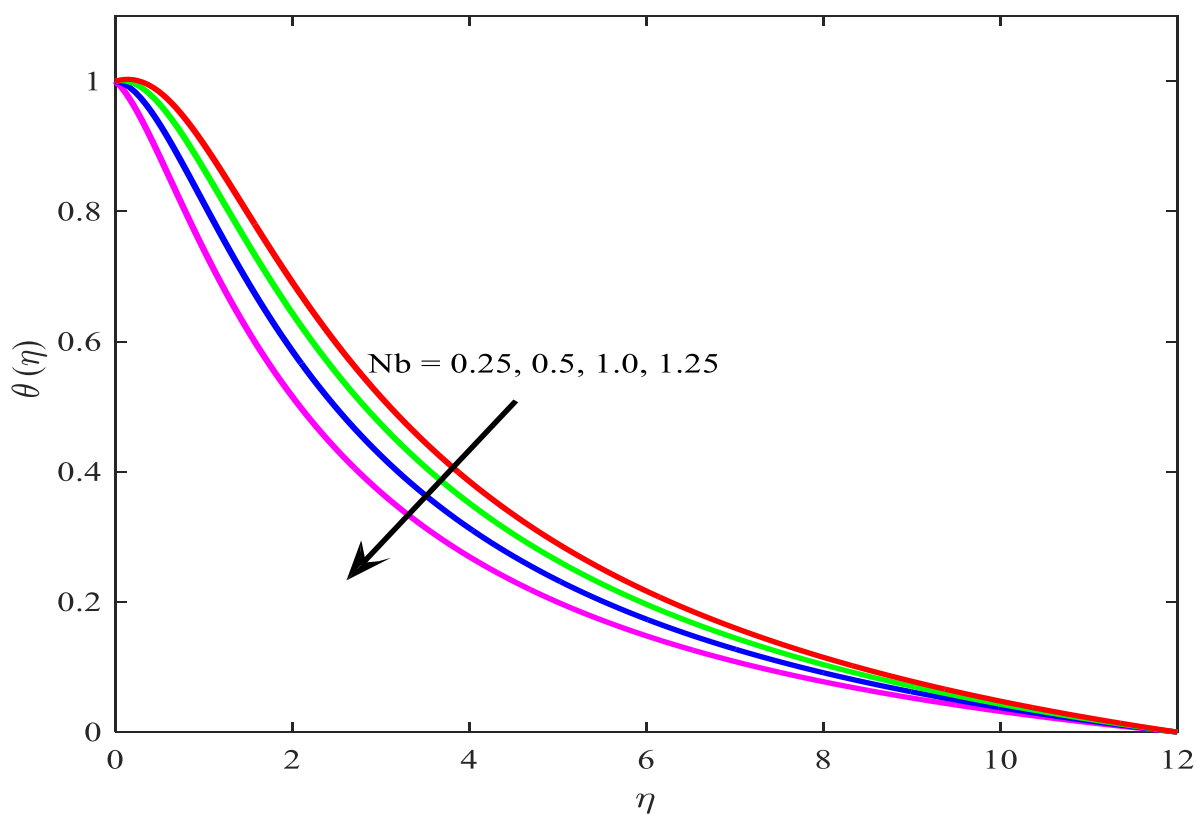

Figure 10. Temperature description, $\theta(\eta)$ for different values of $N b$, where $M=1, N t=0.2, \gamma=1$, $L e=5, L b=0.5, P e=0.5, E c=0.1, \sigma=0.2, \operatorname{Pr}=0.71$.

Figures 13-15 illustrate the $\theta(\eta), \phi(\eta)$ and $\chi(\eta)$ profiles for various values of $N t$. The phenomenon of particle diffusion under the effect of a temperature gradient is called thermophoresis. The force that deposits nanoparticle into the ambient fluid due to the temperature gradient is called thermophoretic force. An increase in thermophoretic force results in the deeper migration of nanoparticles in the ambient fluid. The temperature, concentration, microorganism profiles, and the boundary layer thickness also increase for escalating values of $N t$. This is due to the fact that the magnitude of the temperature gradient at the surface decreases as $N t$ increases. This leads to a condensed thermal boundary layer, and a localized rise in the fluid temperature occurs. 


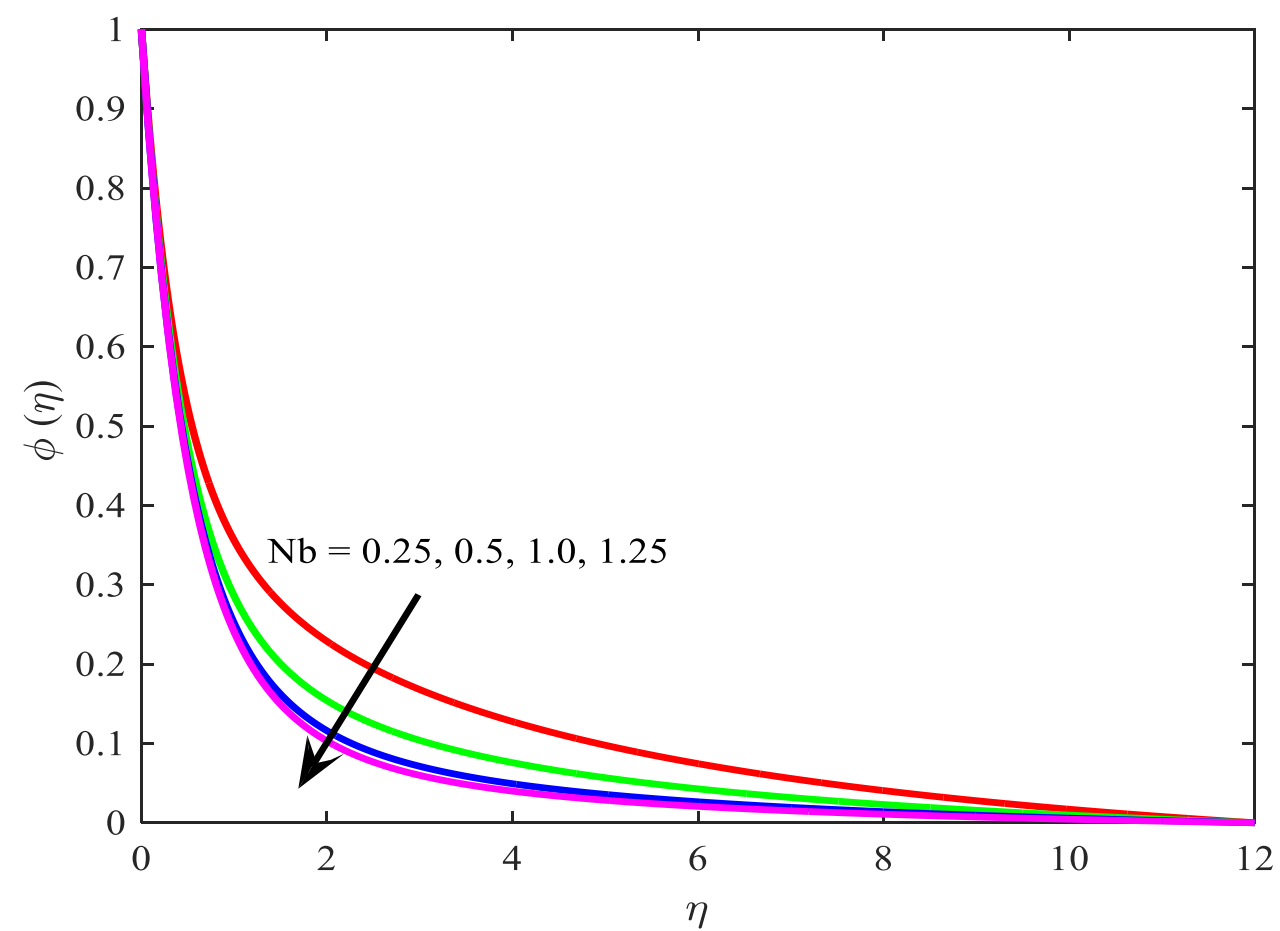

Figure 11. Concentration description, $\phi(\eta)$ for different values of $N b$, where $M=1, N t=0.2, \gamma=1$, $L e=5, L b=0.5, P e=0.5, E c=0.1, \sigma=0.2, \operatorname{Pr}=0.71$.

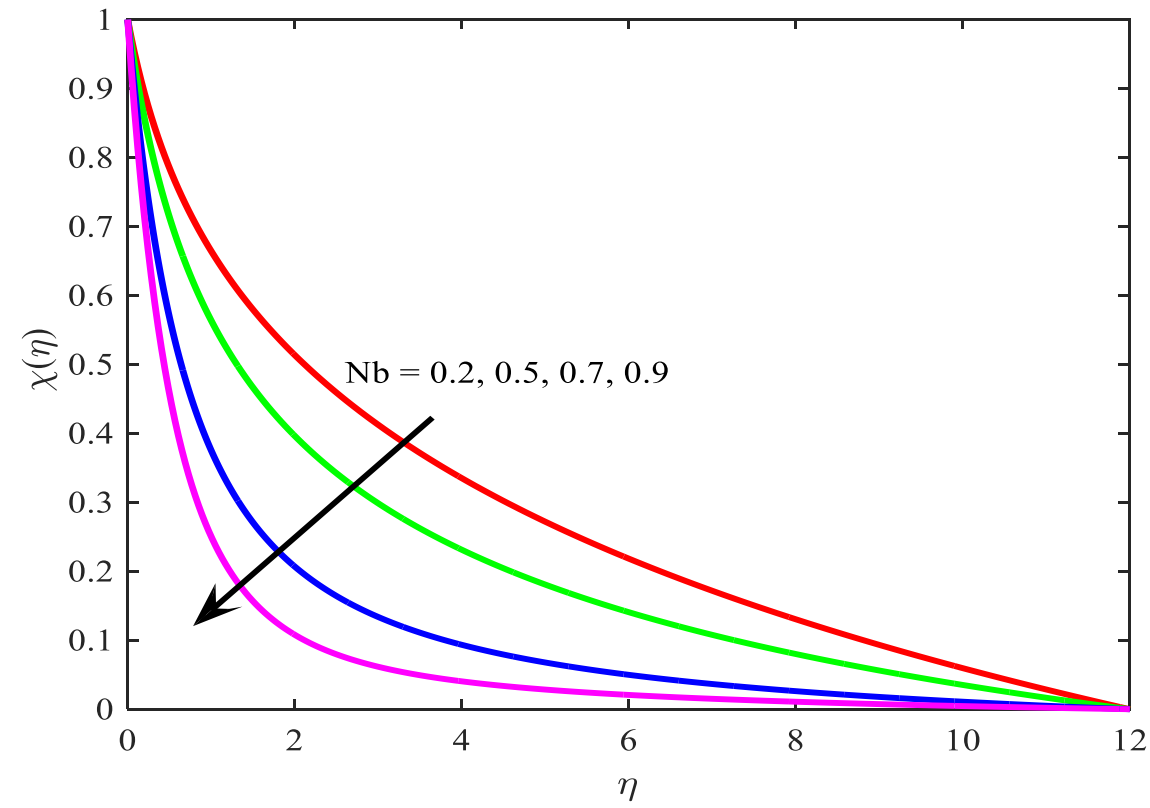

Figure 12. Volume fraction for gyrotactic microorganism profiles, $\chi(\eta)$ for various values of $\mathrm{Nb}$, where $M=1, N t=0.2, \gamma=1, L e=5, L b=0.5, P e=0.5, E c=0.1, \sigma=0.2, \operatorname{Pr}=0.71$.

Figures 16 and 17 describe the species and density of the motile microorganism profile for various parameters of $L e$. We can observe that rising values of Le result in decreasing concentration and microorganism profiles. The Lewis number is a dimensionless number and is defined as the ratio of momentum diffusivity and mass diffusivity. Whenever momentum and mass diffusion convection processes exist, the Lewis number is used to characterize fluid flows. It relates to the relative thickness of the hydrodynamic layer and the mass transfer boundary layer. Rising values of Le indicate strong molecular motions, which ultimately enhance the fluid temperature. Fluid with a larger Lewis number 
possesses a weaker Brownian diffusion coefficient, which causes particles to diffuse deeply into the fluid. Because of this, a shorter penetration depth of temperature exists in the case of higher values of the Lewis number. It illustrates that the dimensionless concentration and motile microorganism profile decline as the Lewis number increases.

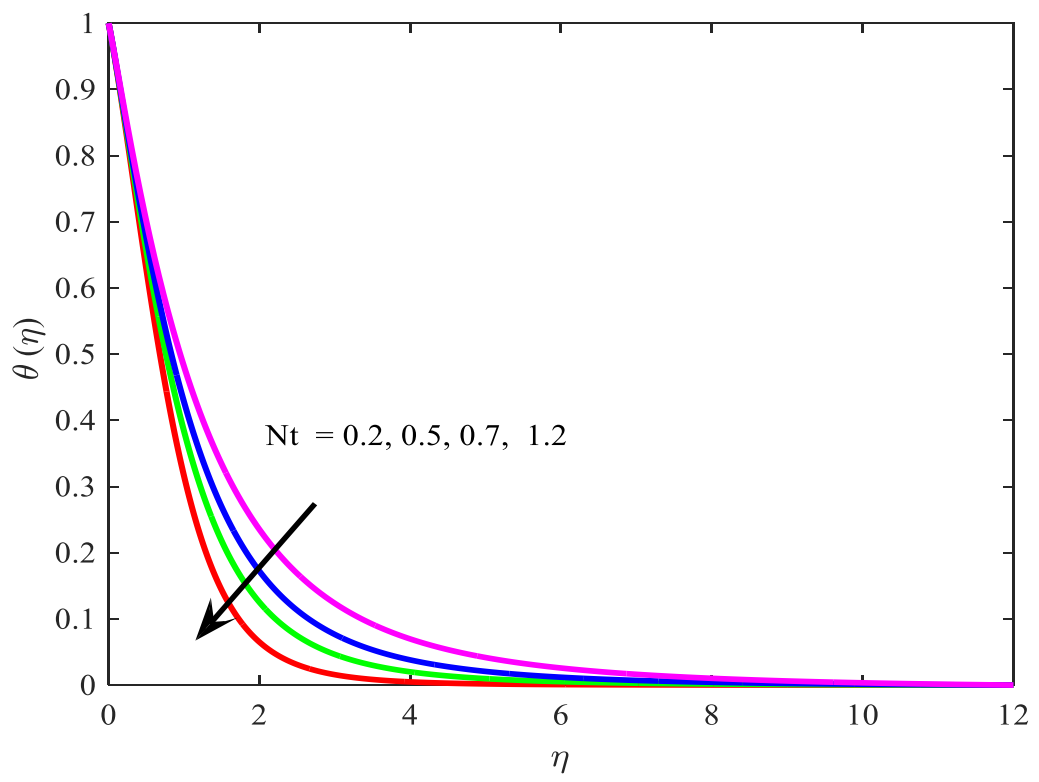

Figure 13. Temperature description, $\theta(\eta)$ for different values of $N t$, where $M=1, \gamma=0.5, N b=0.5$, $L e=5, L b=0.5, P e=0.3, E c=0.1, \sigma=0.2, \operatorname{Pr}=0.72$.

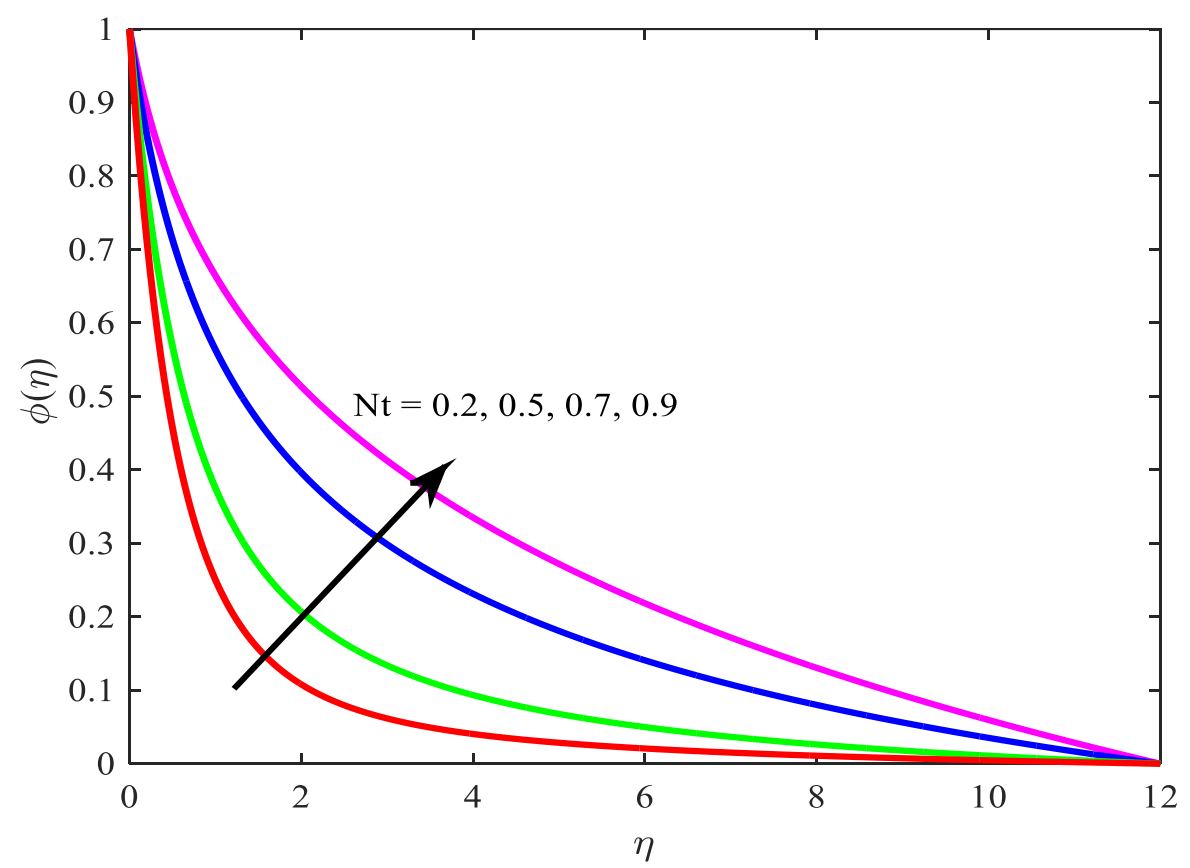

Figure 14. Concentration description, $\phi(\eta)$ for different values of $N t$, where $M=1, \gamma=0.5, N b=0.5$, $L e=5, L b=0.5, P e=0.3, E c=0.1, \sigma=0.2, \operatorname{Pr}=0.72$.

Figures 18-20 illustrate the motile microorganism profile for $P e, L b$, and $\sigma$. From Figure 18 we can see that with escalating values of $P e$, the motile microorganism profile declines. $P e$ is the ratio between the thermal energy convected to the fluid and the thermal energy conducted within the fluid. We can observe from Figure 19, a rapid decline in the profile because the bioconvection Lewis number 
opposes the motion of the fluid. From Figure 20 we can see that for escalating values of the $\sigma$ motile, the microorganism profile declines.

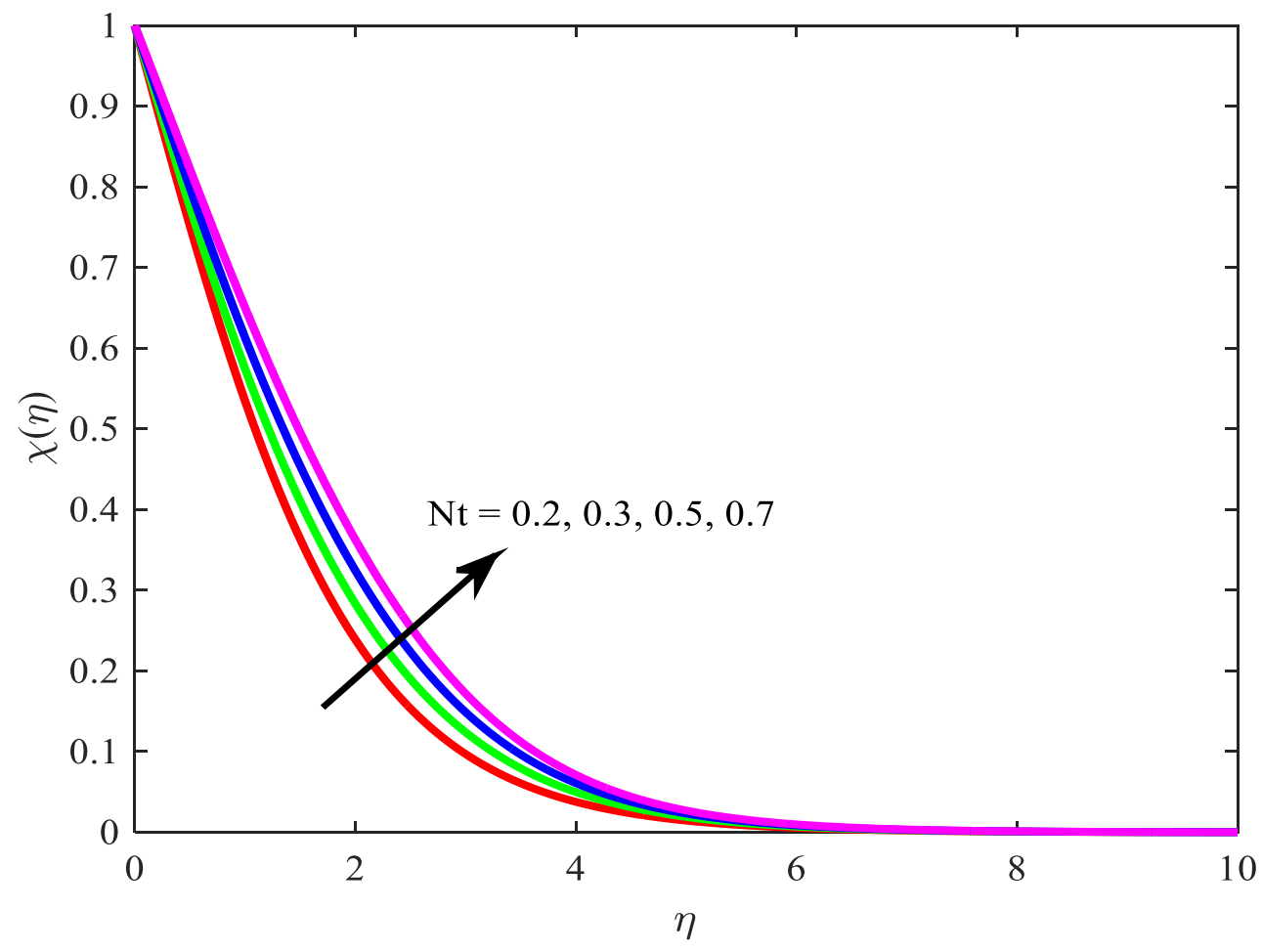

Figure 15. Volume fraction for gyrotactic microorganism description, $\chi(\eta)$ for different values of $N t$, where $M=1, \gamma=0.5, N b=0.5, L e=5, L b=0.5, P e=0.3, E c=0.1, \sigma=0.2, \operatorname{Pr}=0.72$.

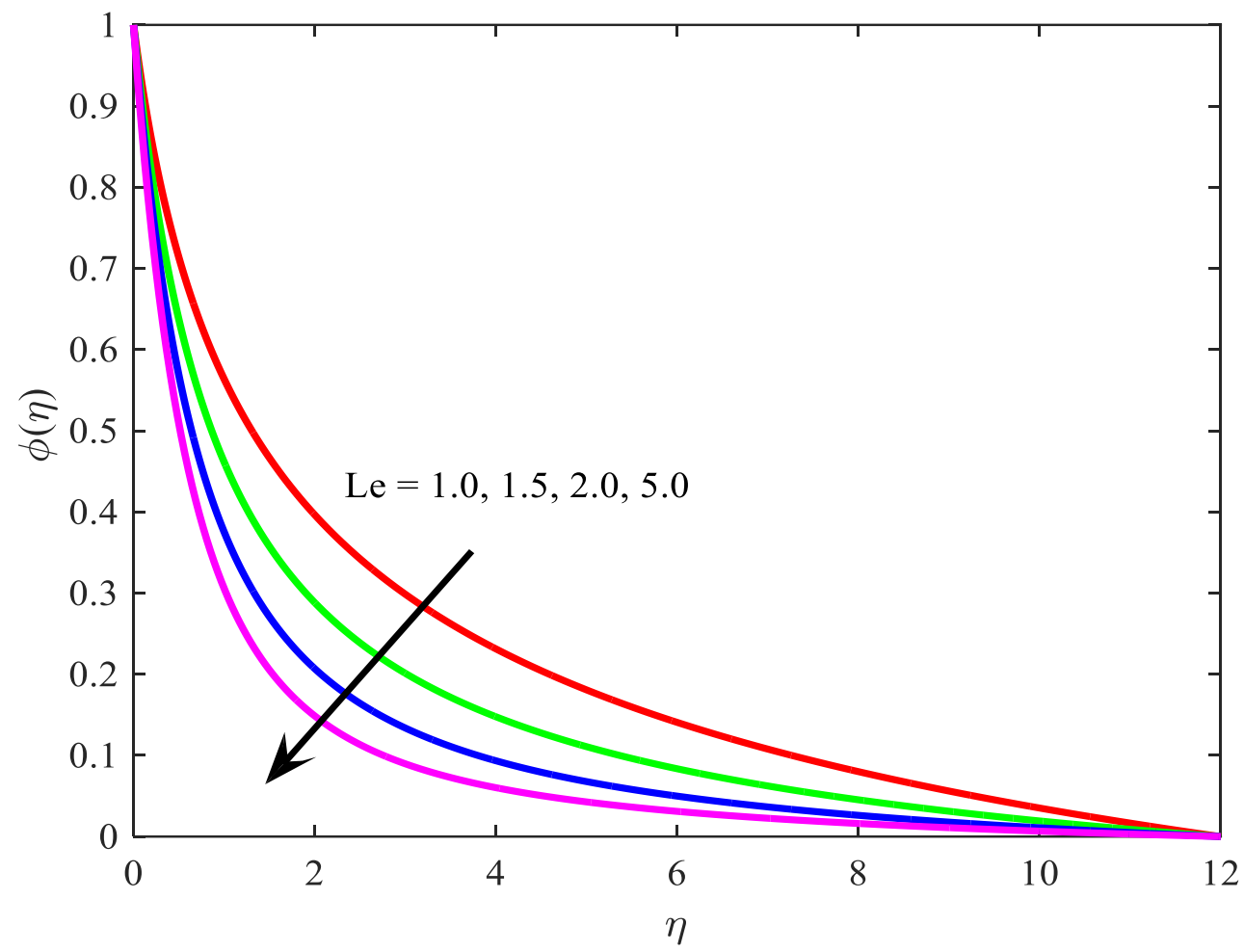

Figure 16. Concentration description, $\phi(\eta)$ for different values of $L e$, where $M=1, N t=0.1, N b=0.5$, $\gamma=1, L b=0.5, P e=0.5, E c=0.2, \sigma=0.2, \operatorname{Pr}=0.72$. 


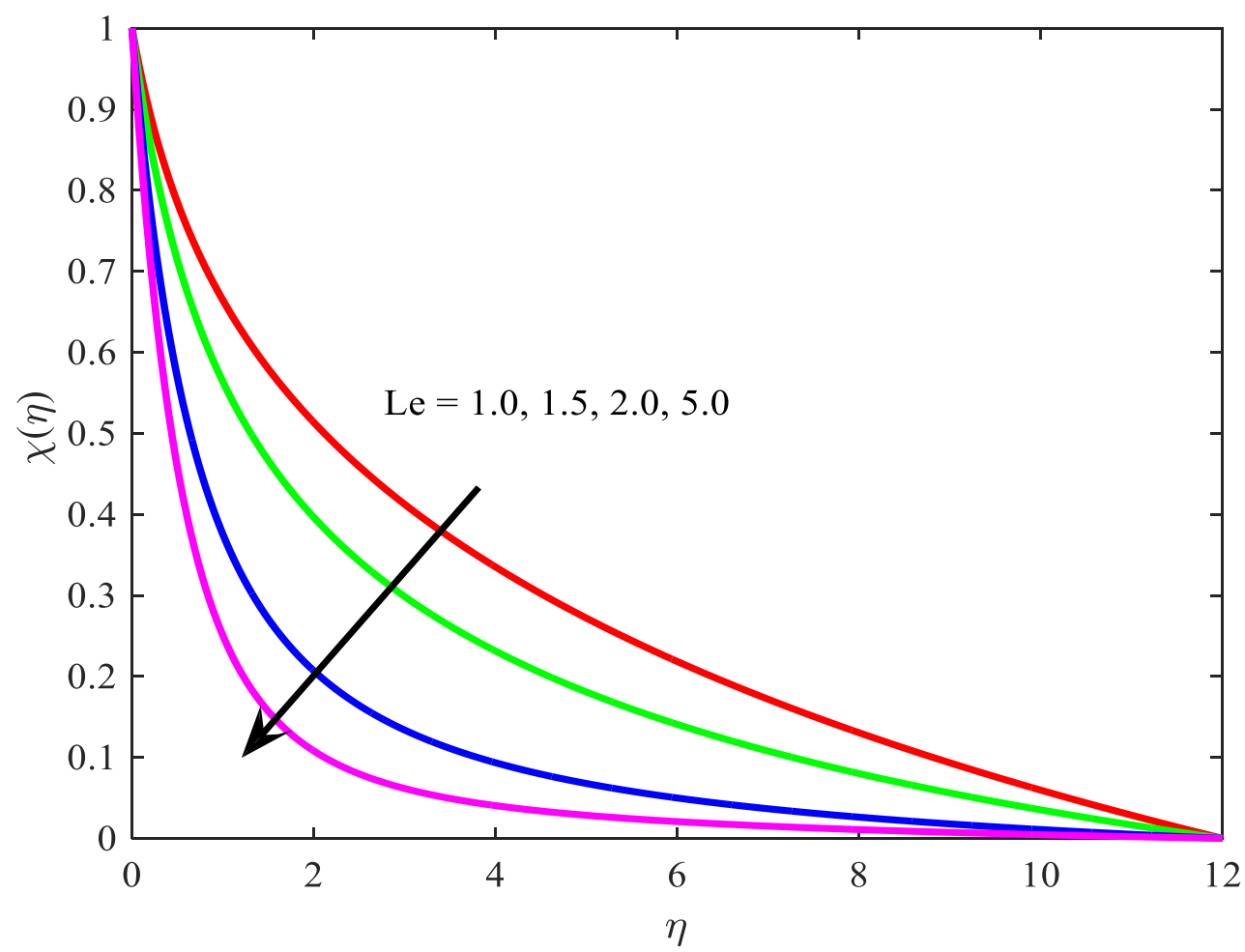

Figure 17. Volume fraction for gyrotactic microorganism profiles, $\chi(\eta)$ for various values of $L e$, where $M=1, N t=0.1, N b=0.5, \gamma=1, L b=0.5, \operatorname{Pe}=0.5, E c=0.2, \sigma=0.2, \operatorname{Pr}=0.72$.

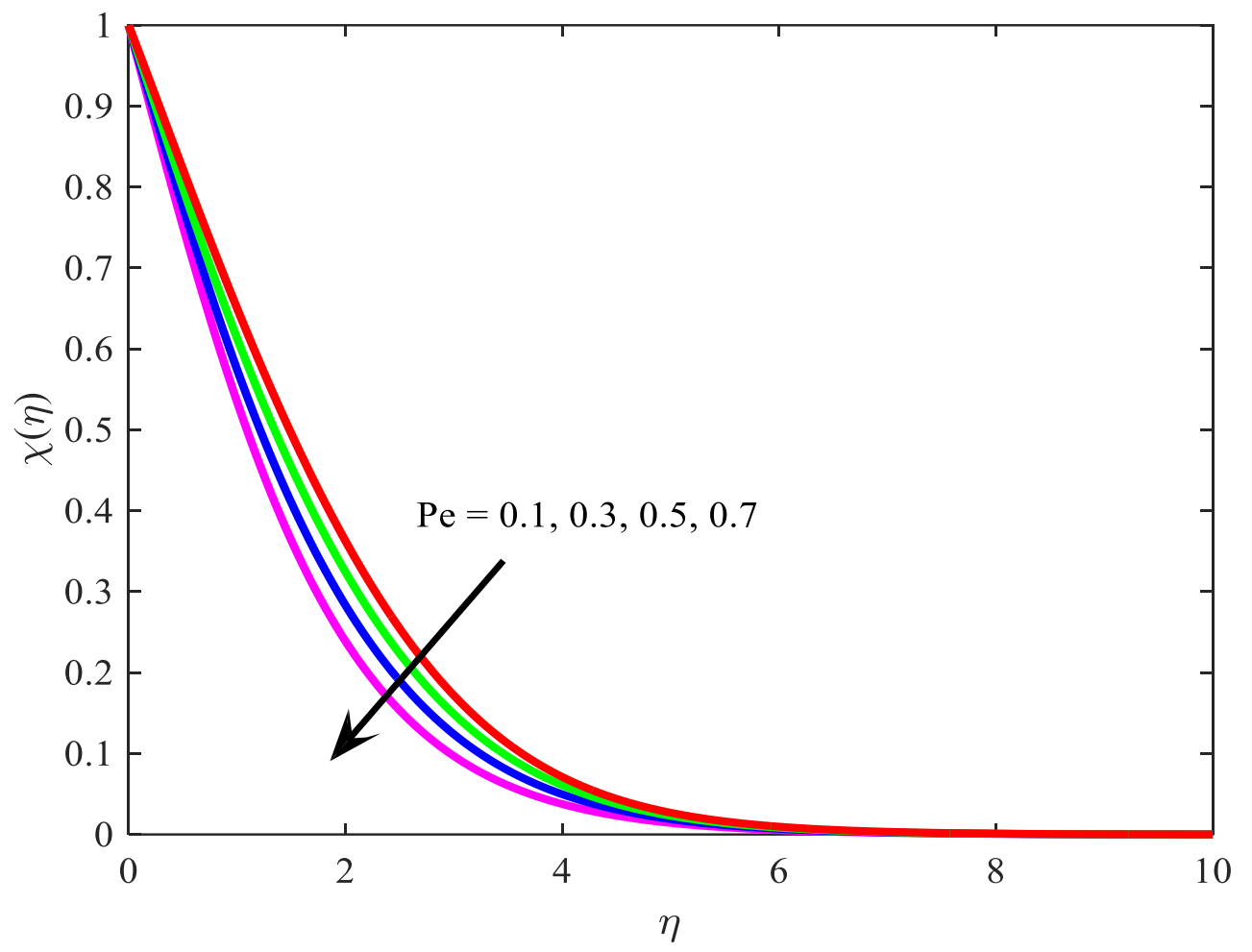

Figure 18. Volume fraction for gyrotactic microorganism profiles, $\chi(\eta)$ for different values of $P e$, where $M=0.5, N t=0.1, N b=0.5, L e=5, L b=0.5, \sigma=0.2, E c=0.2, \gamma=1, \operatorname{Pr}=0.71$. 


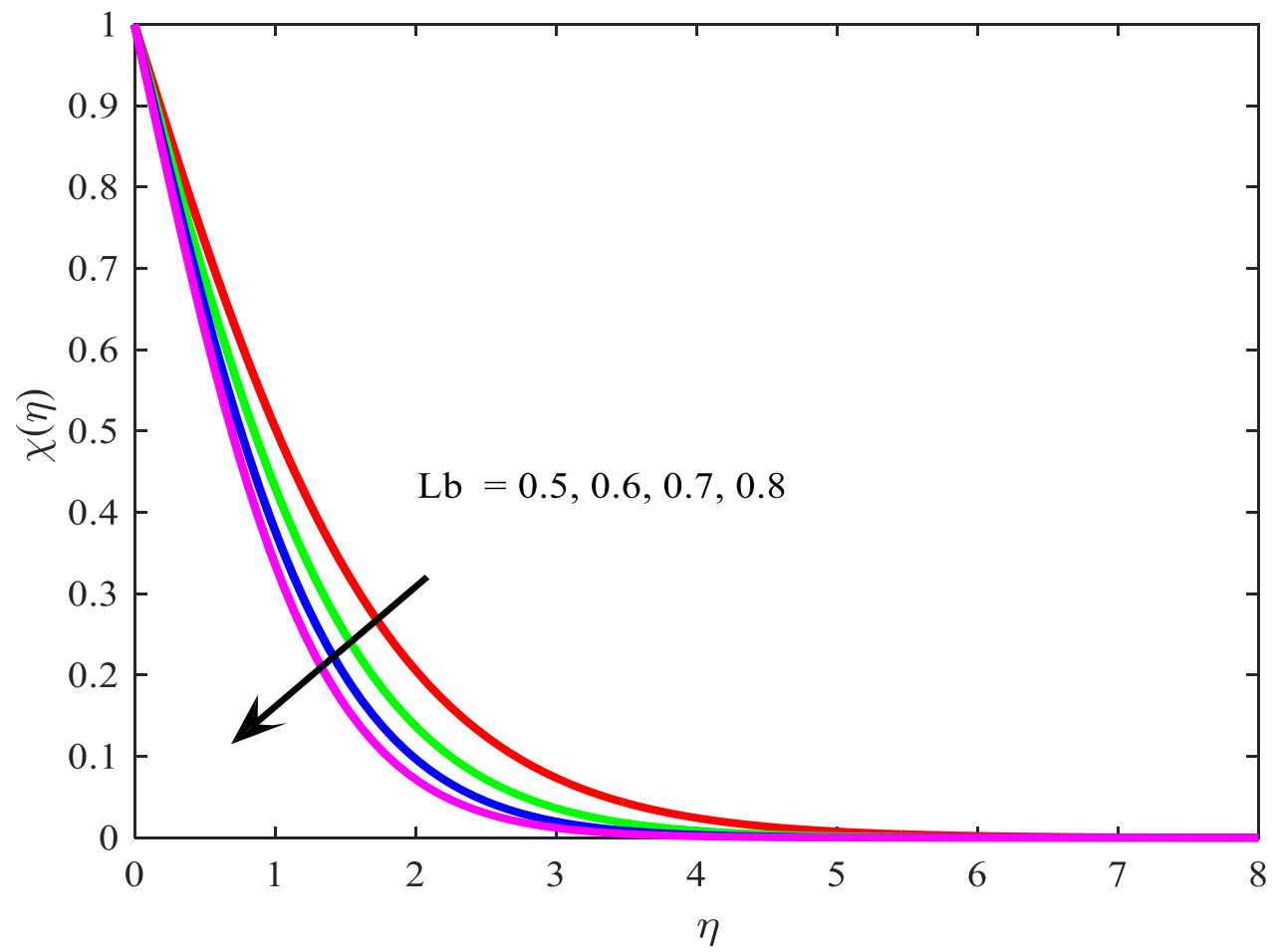

Figure 19. Volume fraction for gyrotactic microorganism profiles, $\chi(\eta)$ for different values of $L b$, where $M=1, N t=0.1, N b=0.5, L e=5, \gamma=1, P e=0.5, E c=0.1, \sigma=0.2, \operatorname{Pr}=0.72$.

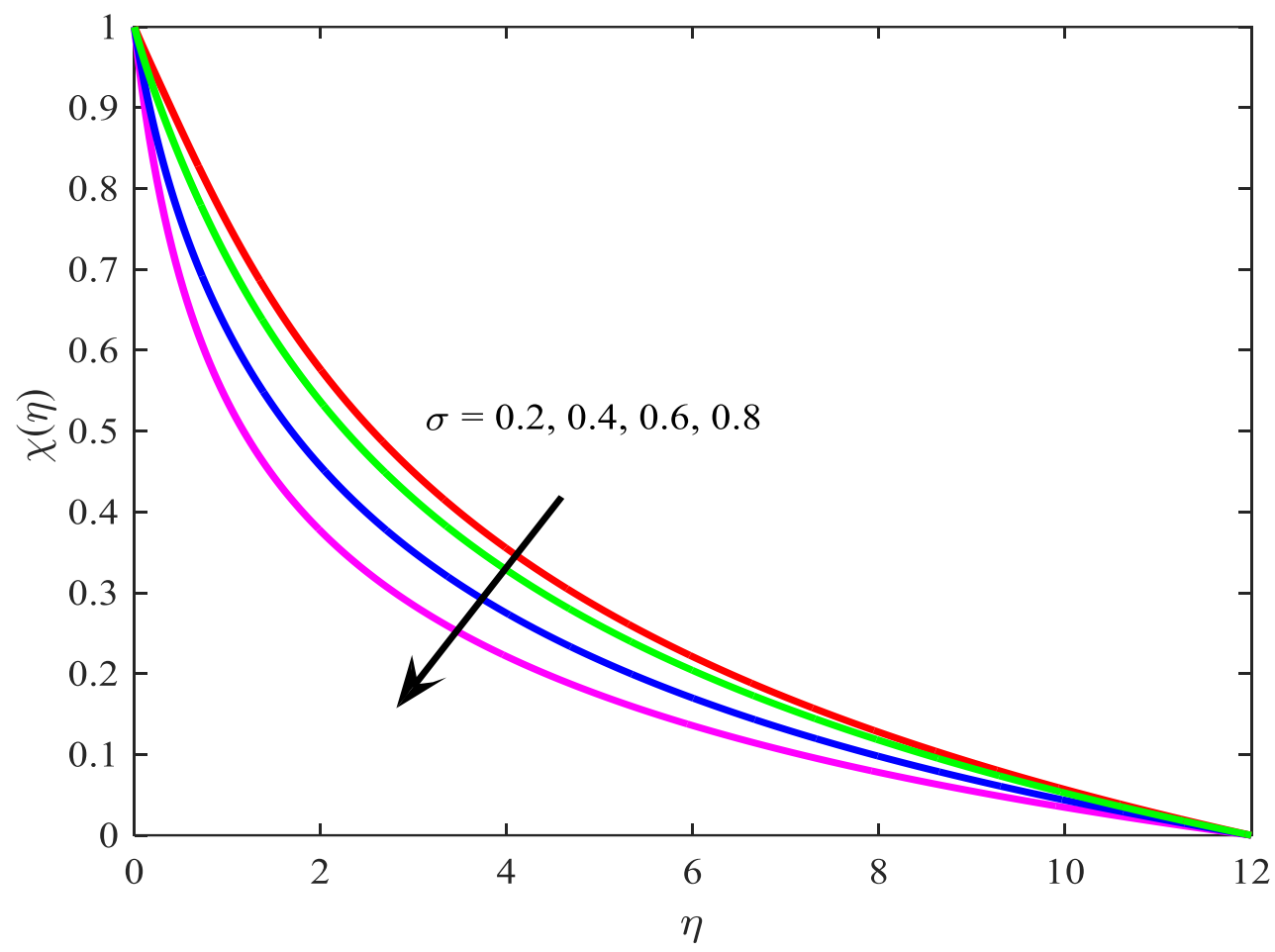

Figure 20. Volume fraction for gyrotactic microorganism profiles, $\chi(\eta)$ for different values of $\sigma$, where $M=1, N t=0.1, N b=0.5, L e=5, \gamma=1, P e=0.5, E c=0.1, \sigma=0.2, \operatorname{Pr}=0.72$.

Figures 21-23 describe the $\theta(\eta), \phi(\eta)$, and $\chi(\eta)$ description for different values of $E c$. A cross-over is found in the concentration and microorganism profile boundary layer at $\eta \approx 0.8$, as shown in Figures 22 and 23. For the dynamic region, $\eta<0.8$, the increase in Ec leads to a reduction in the 
concentration and microorganism boundary layers. On the other hand, for $\eta>0.8$ the reverse takes place as the Eckert number increases. From Figure 21, it can be easily observed that the temperature profile increases with the rise of the $E c$.

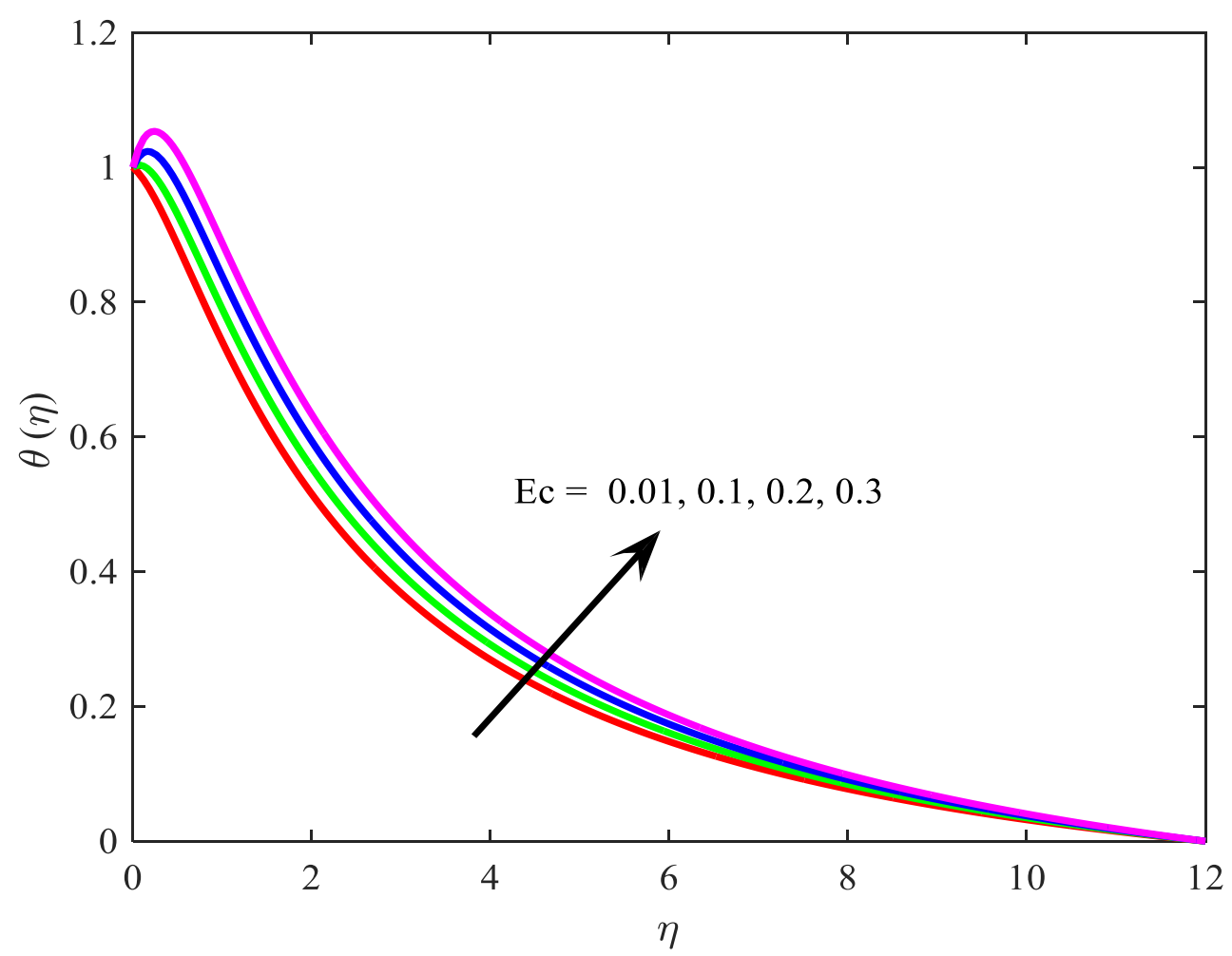

Figure 21. Temperature profiles, $\theta(\eta)$ for different values of $E c$, where $M=1, N t=0.2, N b=0.5$, $L e=5, L b=0.5, P e=0.5, \gamma=1, \sigma=0.2, \operatorname{Pr}=6.17$.

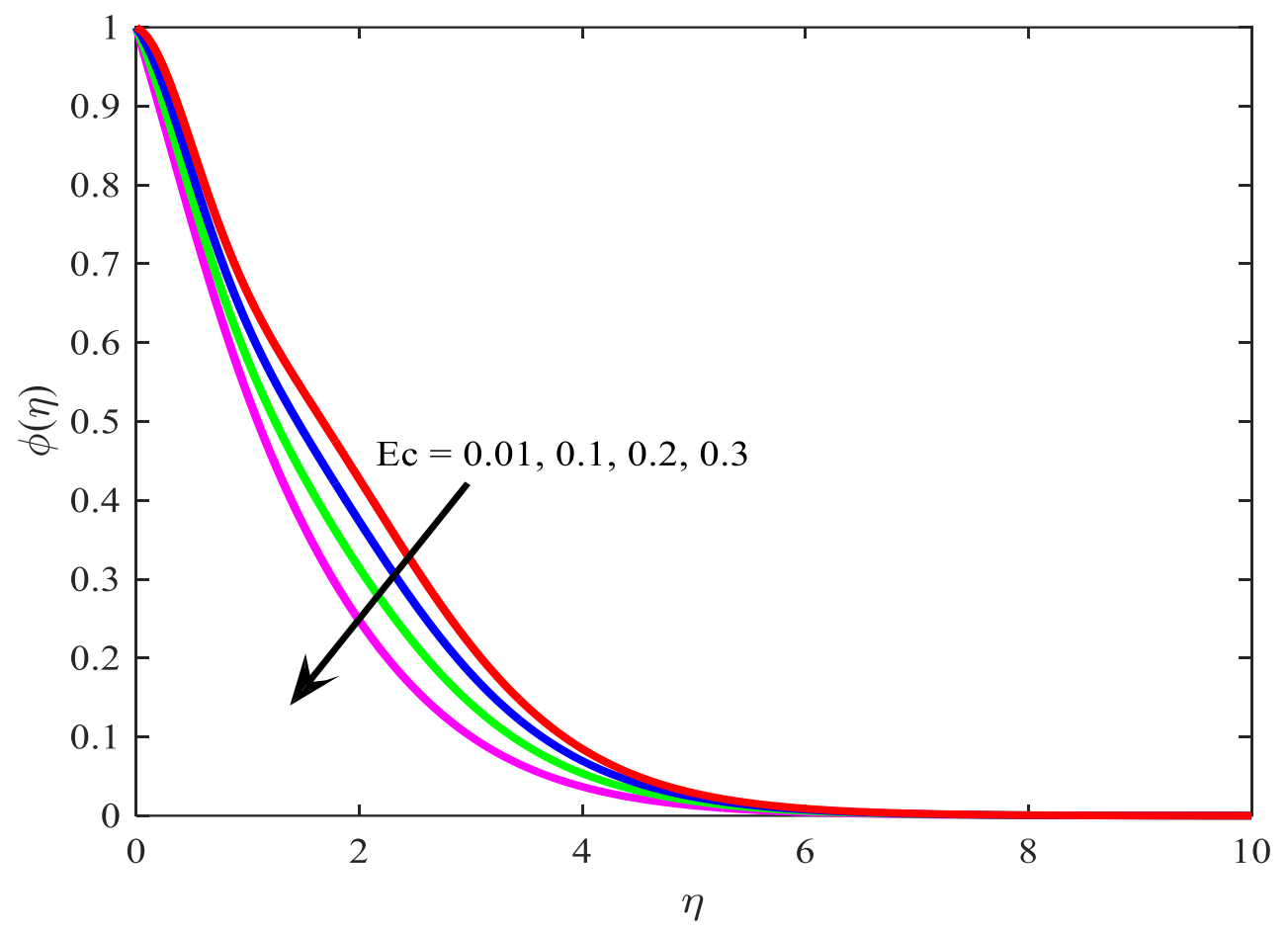

Figure 22. Concentration profiles, $\phi(\eta)$ for different values of $E c$, where $M=1, N t=0.2, N b=0.5$, $L e=5, L b=0.5, P e=0.5, \gamma=1, \sigma=0.2, \operatorname{Pr}=6.17$. 


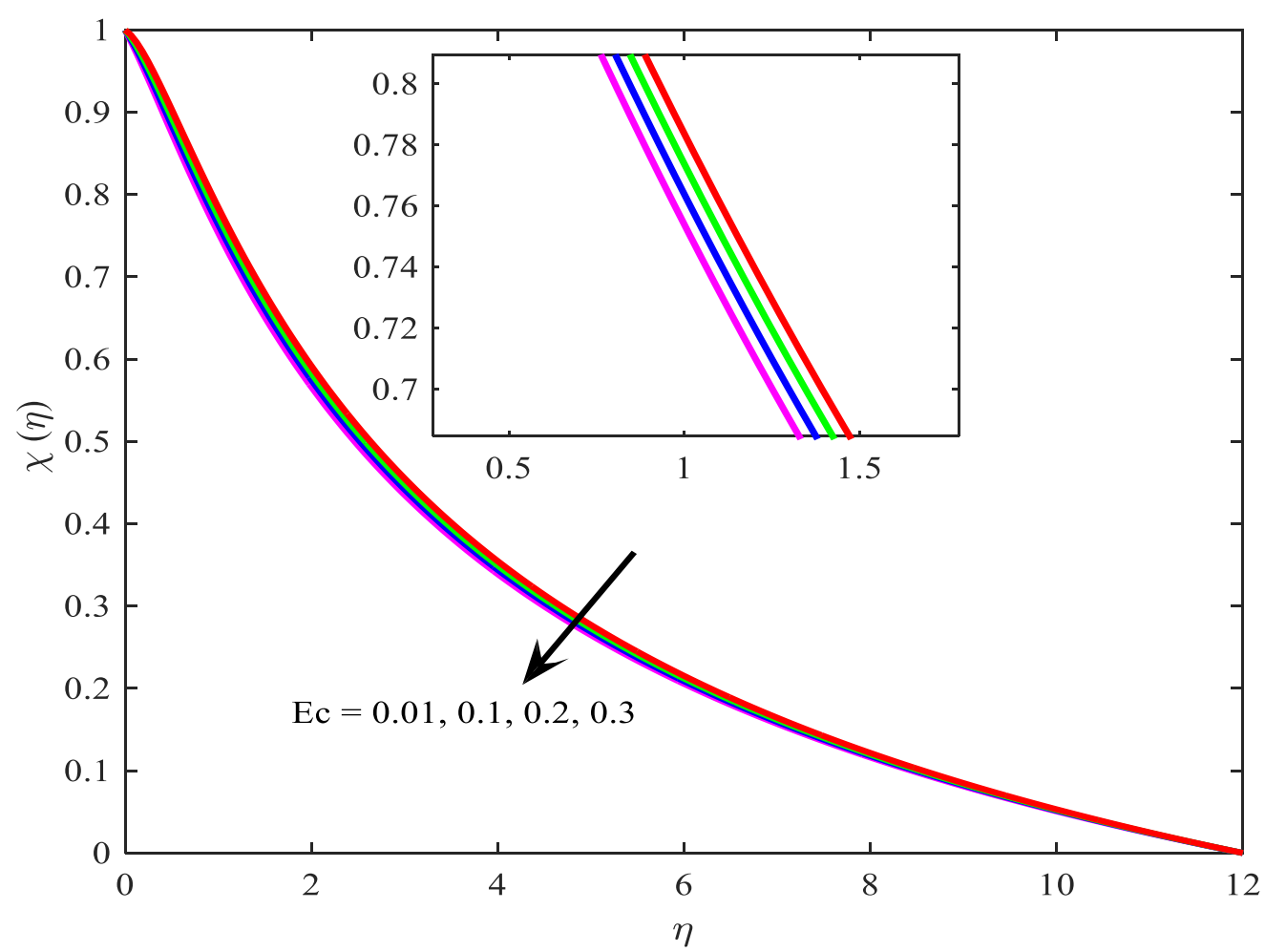

Figure 23. Volume fraction for gyrotactic microorganism profiles, $\chi(\eta)$ for different values of $E c$, where $M=1, N t=0.2, N b=0.5, L e=5, L b=0.5, P e=0.5, \gamma=1, \sigma=0.2, \operatorname{Pr}=6.17$.

Figures 24-26 illustrate the $\theta(\eta), \phi(\eta)$ and $\chi(\eta)$ profiles for different values of Pr. For small values of $\mathrm{Pr}$, the rate of heat transfer and the density number of the microorganism are both intensified, and the rate of mass transfer is decreased, while the revere takes place for higher values of Pr. A cross-over is observed in the nanofluid concentration boundary layer as presented in Figure 25. An increase in $\mathrm{Pr}$ leads to an increase in the concentration boundary layers slightly. Far from the surface, the opposite takes place.

Finally, to get a clear view of the flow field patterns, the streamline dynamics are reported in Figures 27-30, with particular values of the magnetic parameter $M$ and curvature parameter $\gamma$. Where the magnetic parameter $M$ and the curvature parameter $\gamma$ are strengthened, the fluid particle traces a definite curve along the $\mathrm{x}$-direction where the surface is present, but it is clear that there is a retardation in the flow pattern.

The effects of pertinent variables on the physical quantities of $-f^{\prime \prime}(0),-\theta^{\prime}(0),-\phi^{\prime}(0)$, and $-\chi^{\prime}(0)$ are shown in Table 2. It is revealed that for escalating values of $\gamma$, the friction factor increases. We can fix the value of $\gamma=1.0$ and the remaining parameter values are changed. $-f^{\prime \prime}(0),-\theta^{\prime}(0),-\phi^{\prime}(0)$, and $-\chi^{\prime}(0)$ were the same, and we also observe that the friction factor $-f^{\prime \prime}(0)$ increases to the higher values of $\gamma$ and $M$. The rate of the heat transfer coefficient, $-\theta^{\prime}(0)$ increases for the mounting values of $\gamma, \operatorname{Pr}, N b$, whereas the opposite is true for the remaining other flow variables. The rate of the species transfer coefficient, $-\phi^{\prime}(0)$ declines with the strengthening of $\gamma, M, \operatorname{Pr}, N t$, while the other parameters show the reverse behavior. Finally, the rate of motile microorganism $-\chi^{\prime}(0)$ is more for $\gamma, \mathrm{Pr}, \mathrm{Pe}, \mathrm{Lb}$. From Table 2, it is clear that the surface shear stress is not affected by any modification of $\mathrm{Nb}$, so nanofluid velocity is not affected either. The friction factor is not affected by any variation of $N t$, and it was also observed that the surface shear stress and Nusselt number are not influenced by any change of $L e$, so the nanofluid velocity and temperature are not affected either. 


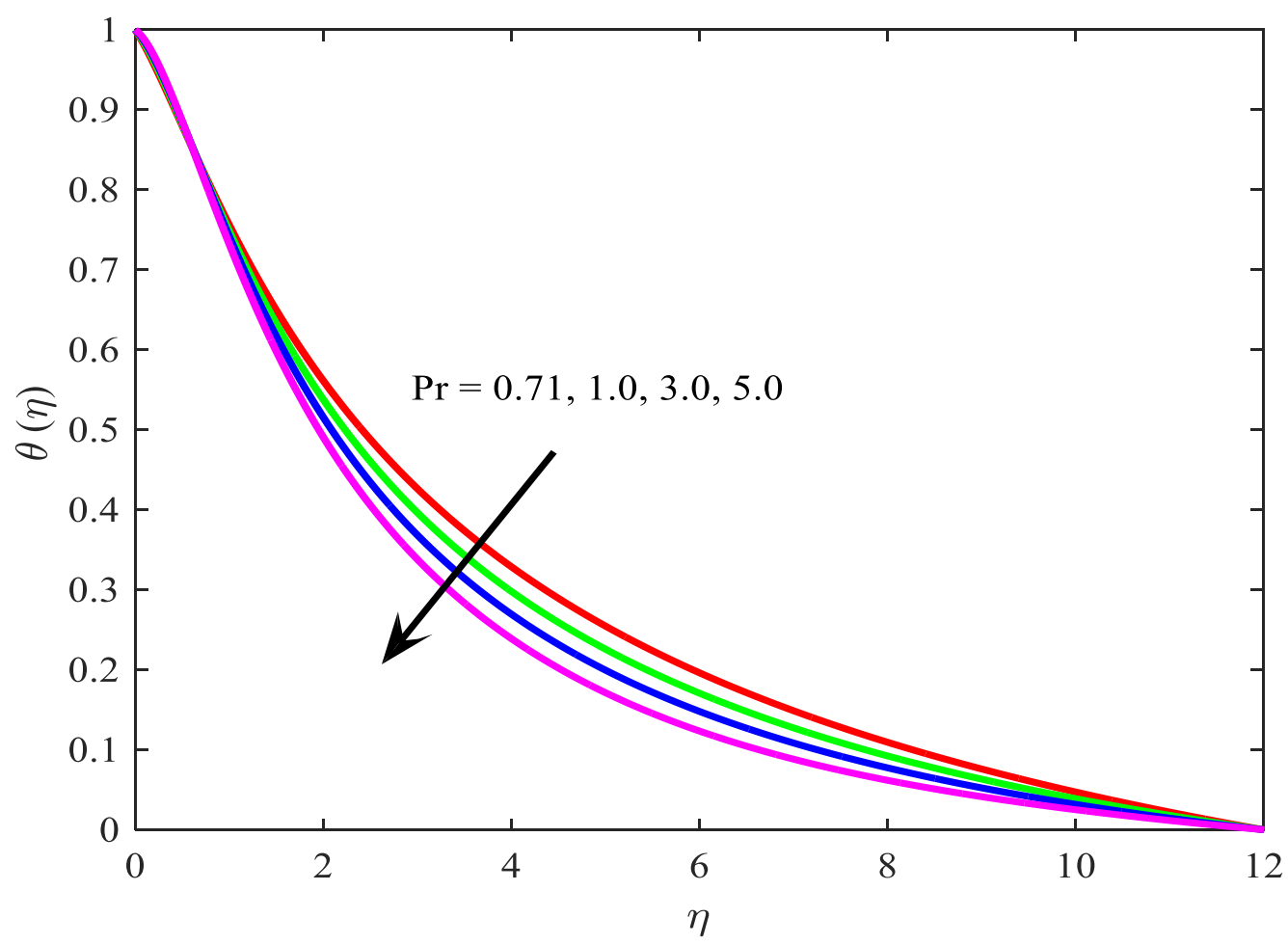

Figure 24. Temperature profiles, $\theta(\eta)$ for different values of $\operatorname{Pr}$, where $M=1, N t=0.2, N b=0.5$, $L e=5, L b=0.5, P e=0.5, E c=0.1, \sigma=0.2, \gamma=1$.

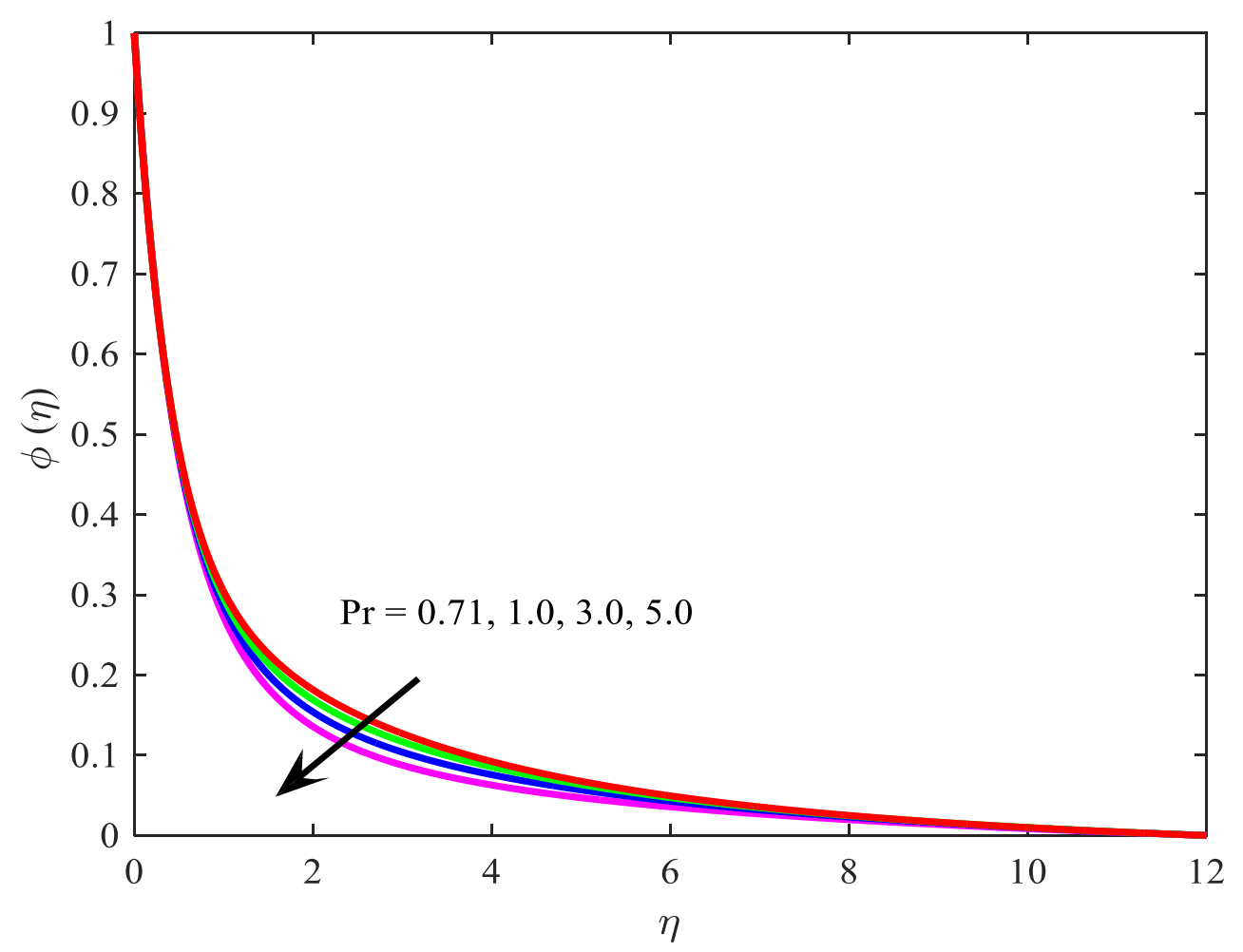

Figure 25. Concentration profiles, $\phi(\eta)$ for different values of $\operatorname{Pr}$, where $M=1, N t=0.2, N b=0.5$, $L e=5, L b=0.5, P e=0.5, E c=0.1, \sigma=0.2, \gamma=1$. 


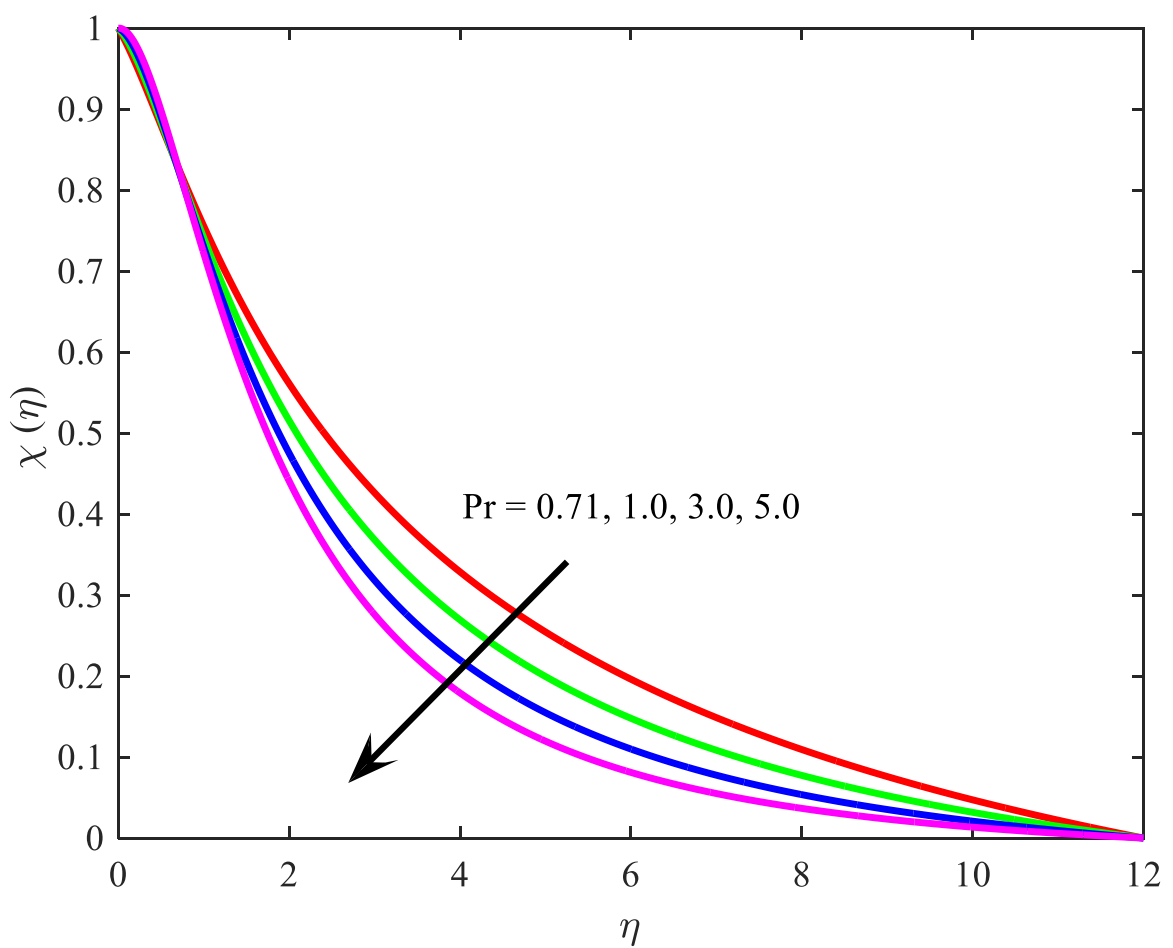

Figure 26. Volume fraction for gyrotactic microorganism profiles, $\chi(\eta)$ for different values of $\mathrm{Pr}$, where $M=1, N t=0.2, N b=0.5, L e=5, L b=0.5, P e=0.5, E c=0.1, \sigma=0.2, \gamma=1$.

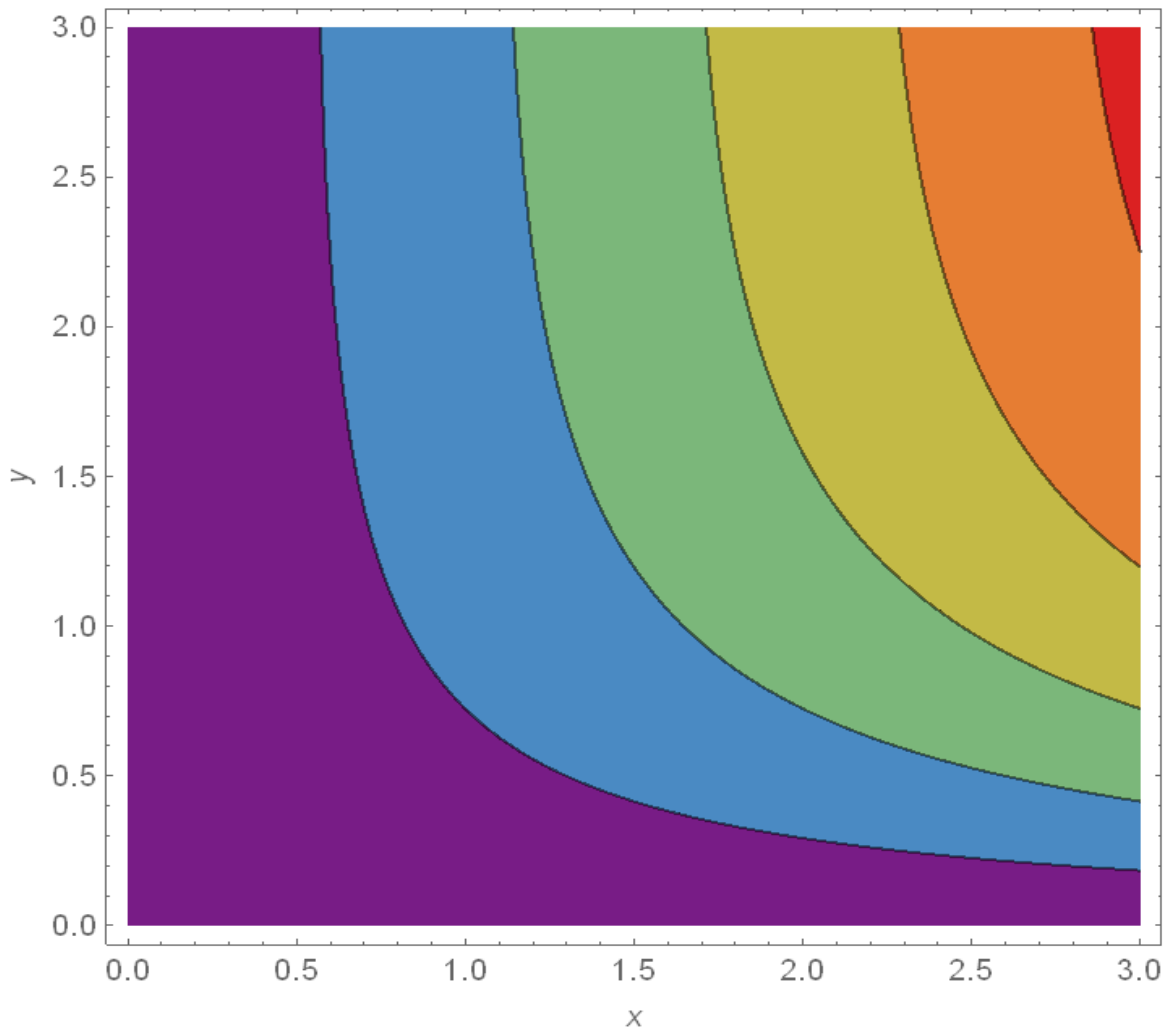

Figure 27. Streamlines for $\gamma=0.1, M=0.0$. 


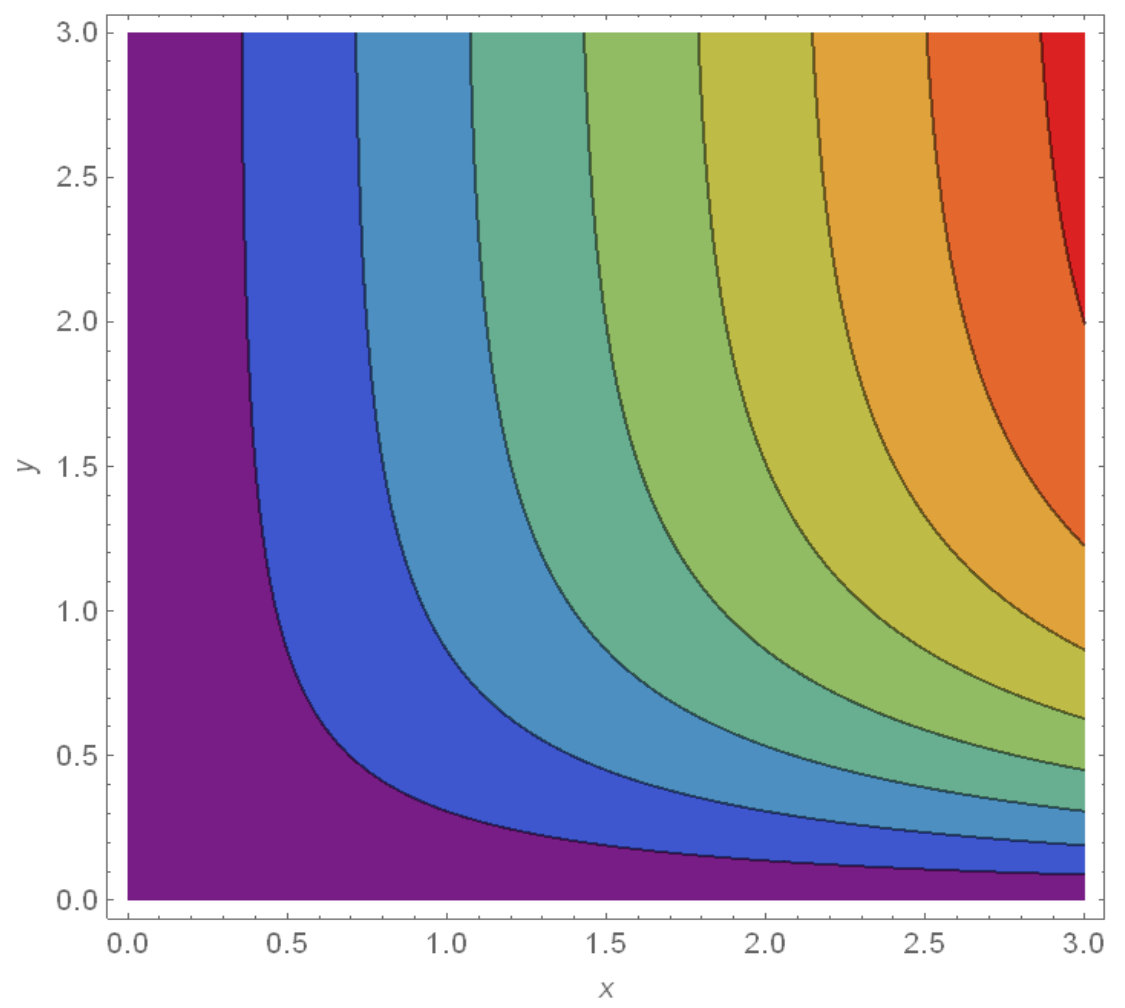

Figure 28. Streamlines for $\gamma=0.3, M=0.5$.

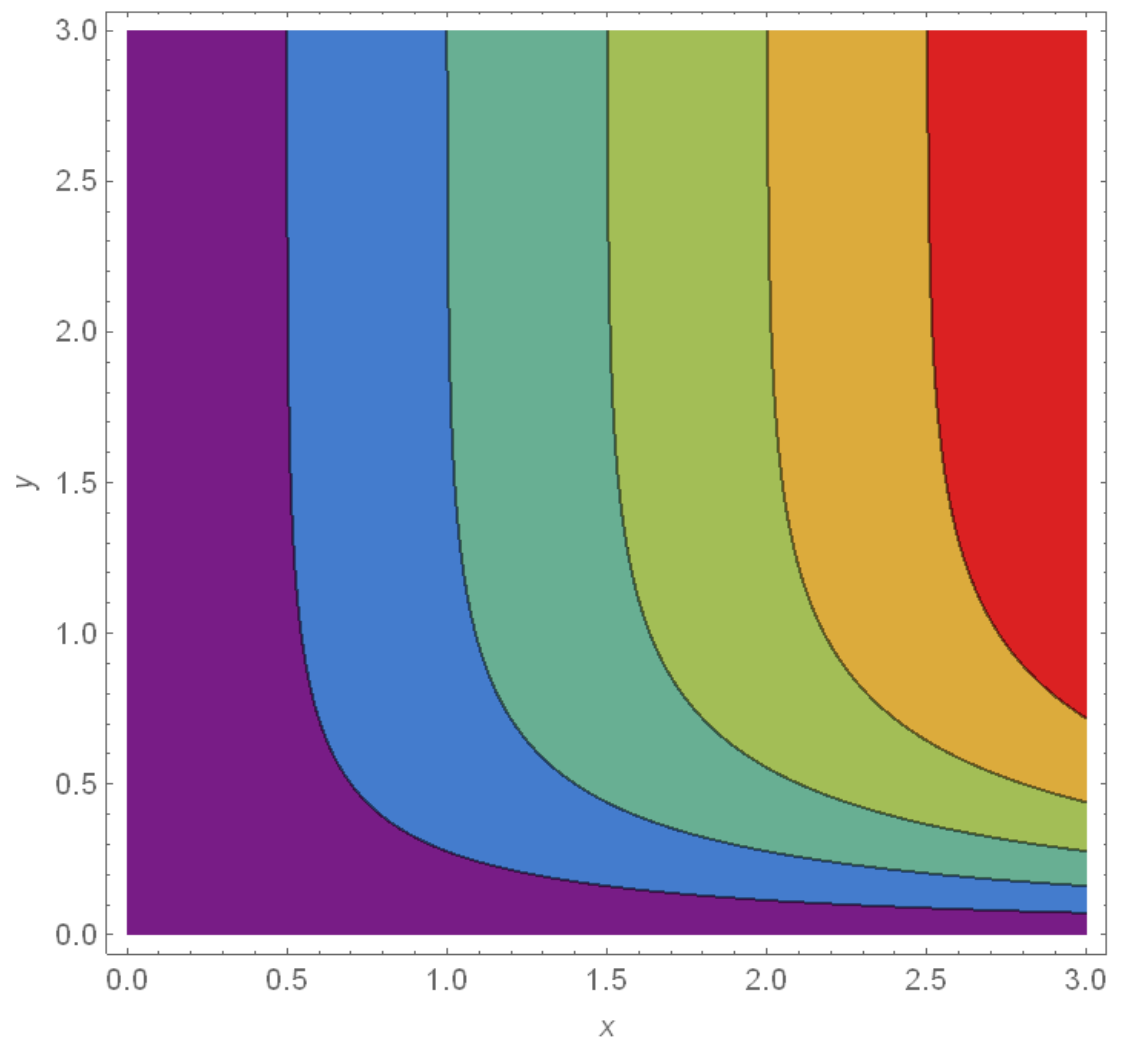

Figure 29. Streamlines for $\gamma=1.0, M=1.5$. 


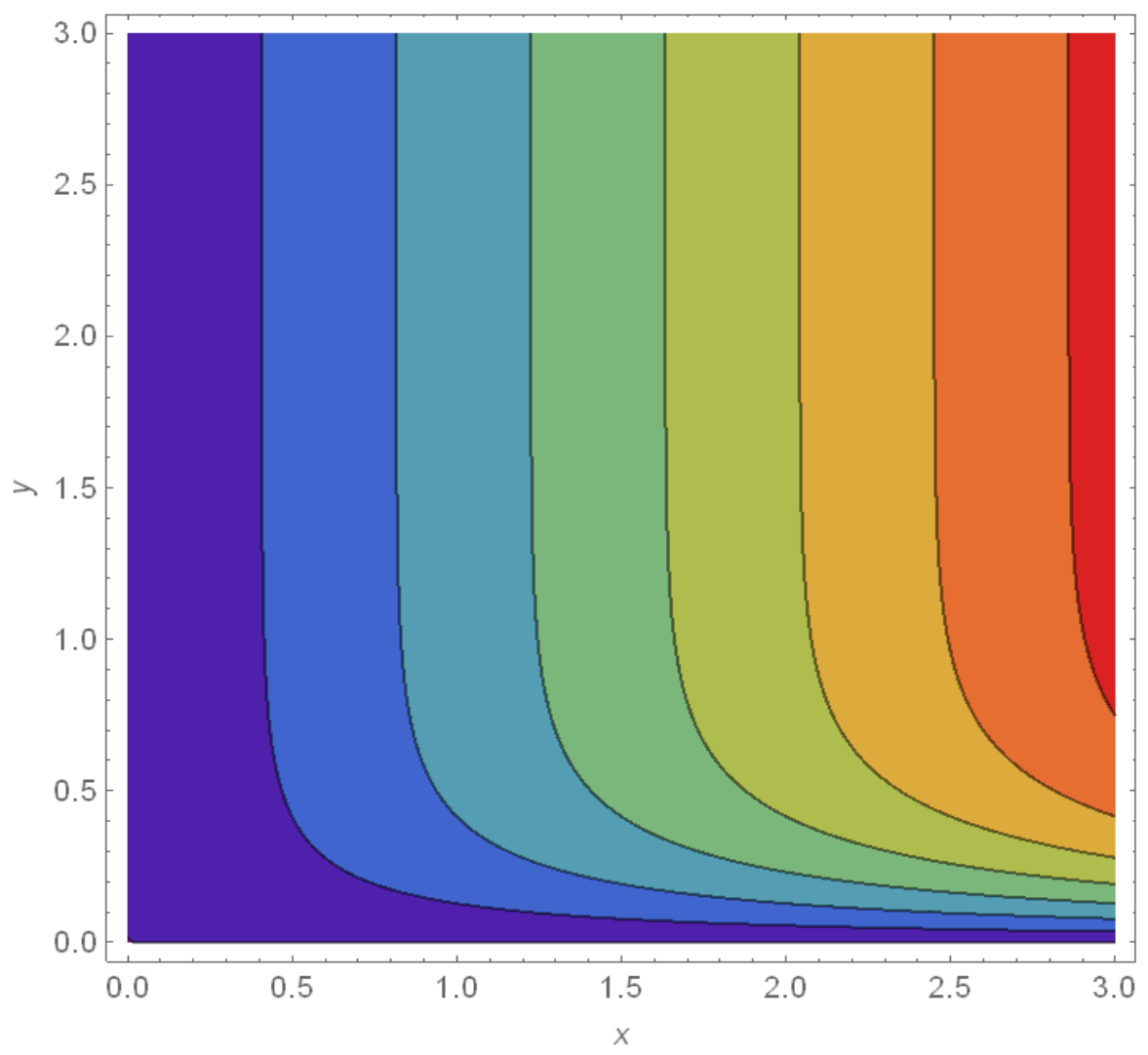

Figure 30. Streamlines for $\gamma=2.0, M=6.0$. 
Table 2. $M, N t, N b, L e, L b, P e, E, \sigma, \operatorname{Pr}, \gamma$ on the cylinder surface physical quantities, $-f^{\prime \prime}(0),-\theta^{\prime}(0),-\phi^{\prime}(0)$ and $-\chi^{\prime}(0)$.

\begin{tabular}{|c|c|c|c|c|c|c|c|c|c|c|c|c|c|}
\hline$\gamma$ & $M$ & Pr & $P e$ & $N b$ & $N t$ & Le & $L b$ & $\sigma$ & $E c$ & $-f^{\prime \prime}(0)$ & $-\boldsymbol{\theta}^{\prime}(0)$ & $-\phi^{\prime}(0)$ & $-\chi^{\prime}(0)$ \\
\hline 0.5 & \multirow{4}{*}{1} & \multirow{4}{*}{5} & \multirow{4}{*}{0.3} & \multirow{4}{*}{0.5} & \multirow{4}{*}{0.2} & \multirow{4}{*}{5} & \multirow{4}{*}{0.2} & \multirow{4}{*}{0.2} & \multirow{4}{*}{0.01} & 1.62649308 & 2.42810268 & 0.85568261 & 0.77721513 \\
\hline 1 & & & & & & & & & & 1.82111645 & 2.67528648 & 0.81591240 & 0.91919885 \\
\hline 2 & & & & & & & & & & 2.17941349 & 3.03778670 & 0.79123180 & 1.17739872 \\
\hline 3 & & & & & & & & & & 2.51241569 & 3.23014786 & 0.83834565 & 1.43491309 \\
\hline \multirow{3}{*}{1} & 0 & \multirow{3}{*}{0.71} & \multirow{3}{*}{0.5} & \multirow{3}{*}{0.5} & \multirow{3}{*}{0.1} & \multirow{3}{*}{5} & \multirow{3}{*}{0.5} & \multirow{3}{*}{0.2} & \multirow{3}{*}{0.2} & 1.35577051 & 1.08610352 & 1.71241497 & 1.34116551 \\
\hline & 1 & & & & & & & & & 2.82111645 & 0.71283719 & 1.63058296 & 1.28080156 \\
\hline & 2 & & & & & & & & & 2.15890400 & 0.32331590 & 1.59635412 & 1.25335316 \\
\hline \multirow{4}{*}{1} & \multirow{4}{*}{1} & 0.71 & \multirow{4}{*}{0.5} & \multirow{4}{*}{0.5} & \multirow{4}{*}{0.2} & \multirow{4}{*}{5} & \multirow{4}{*}{0.5} & & & 1.82111645 & 0.99942787 & 1.39642364 & 1.12678471 \\
\hline & & 1 & & & & & & & & 1.82111645 & 1.08600254 & 1.37153874 & 1.13654847 \\
\hline & & 5 & & & & & & 0.2 & 0.1 & 1.82111645 & 1.12591585 & 1.40748237 & 1.52920649 \\
\hline & & 7 & & & & & & & & 1.82111645 & 0.87986583 & 1.51248713 & 1.77773396 \\
\hline & & & 0.3 & & & & & & & 1.82111645 & 0.71283719 & 1.63058296 & 1.19404622 \\
\hline 1 & 1 & 0.71 & 0.5 & 0.5 & 0.1 & 5 & 0.5 & 0.2 & 0.2 & 1.82111645 & 0.71283719 & 1.63058296 & 1.28080156 \\
\hline & & & 0.7 & & & & & & & 1.82111645 & 0.71283719 & 1.63058296 & 1.35483099 \\
\hline & & & & 0.25 & & & & & & 1.62649308 & 0.40047131 & 1.39670138 & 0.97004499 \\
\hline 0.5 & 1 & 0.71 & 0.5 & 0.5 & 0.2 & 5 & 0.5 & 0.2 & 0.1 & 1.62649308 & 0.62834887 & 1.43755939 & 0.98733892 \\
\hline & & & & 1.2 & & & & & & 1.62649308 & 1.18153356 & 1.47036457 & 0.99954647 \\
\hline & & & & & 0.2 & & & & & 1.62649308 & 0.81885213 & 1.37025917 & 0.87739871 \\
\hline 0.5 & 1 & 0.71 & 0.3 & 0.5 & 0.5 & 5 & 0.5 & 0.2 & 0.1 & 1.62649308 & 0.72661881 & 1.07378725 & 0.73600200 \\
\hline & & & & & 1.2 & & & & & 1.62649308 & 0.55314537 & 0.63142889 & 0.49242417 \\
\hline & & & & & & 1 & & & & 1.82111645 & 0.71283719 & 0.67841111 & 0.64189010 \\
\hline 1 & 1 & 071 & 0.5 & 05 & 01 & 1.5 & 05 & 02 & $0 ?$ & 1.82111645 & 0.71283719 & 0.81415772 & 0.73312212 \\
\hline 1 & 1 & 0.11 & 0.5 & 0.5 & 0.1 & 2 & 0.5 & 0.2 & 0.2 & 1.82111645 & 0.71283719 & 0.94753387 & 0.81755282 \\
\hline & & & & & & 5 & & & & 1.82111645 & 0.71283719 & 1.63058296 & 1.19404622 \\
\hline & & & & & & & 0.5 & & & 1.82111645 & 1.67403063 & 1.47926112 & 1.55544165 \\
\hline 1 & 1 & 5 & 0.5 & 0.5 & 0.1 & 5 & 0.6 & 0.2 & 0.1 & 1.82111645 & 1.67403063 & 1.47926112 & 1.63930009 \\
\hline & & & & & & & 0.7 & & & 1.82111645 & 1.67403063 & 1.47926112 & 1.7210218 \\
\hline & & & & & & & & 0.2 & & 1.82111645 & 1.04300428 & 1.56883842 & 1.23920758 \\
\hline 1 & 1 & 0.71 & 0.5 & 0.5 & 0.1 & 5 & 0.5 & 0.4 & 0.2 & 1.82111645 & 1.04300428 & 1.56883842 & 1.49489645 \\
\hline & & & & & & & & 0.6 & & 1.82111645 & 1.04300428 & 1.56883842 & 1.69489645 \\
\hline & & & & & & & & & 0.01 & 1.82111645 & 2.80350693 & 0.77232258 & 1.19996973 \\
\hline 1 & 1 & 6.17 & 0.5 & 0.5 & 0.2 & 5 & 0.5 & 0.2 & 0.1 & 1.82111645 & 0.99256106 & 1.46526840 & 1.67326495 \\
\hline & & & & & & & & & 0.2 & 1.82111645 & 0.53315880 & 1.64145133 & 1.79135562 \\
\hline
\end{tabular}




\section{Conclusions}

In the current investigation, we examined boundary layer motion, thermal and species transfer of nanofluid comprising microorganisms across an extending cylinder. The effects of magnetic field, Brownian diffusion, and thermophoresis were taken into account. By employing suitable transformations for momentum, heat, mass, and microorganism, the boundary layer equations of the flow were converted to ODEs and solved by applying the SRM. The impacts of curvature parameter $\gamma$, Peclet number $P e$, Lewis number $L b$, Prandtl number Pr, Eckert number $E c$, Lewis number $L e$, Magnetic parameter $M$, Brownian motion parameter $N b$, thermophoresis parameter $N t$, bioconvection constant $\sigma$, the friction factor, the rate of heat transfer, the rate of mass transfer, and the density number of the motile microorganism have been studied. The results are summarized as follows:

- $\quad M, N t, E c$ enhance the temperature field $\theta$;

- $M$ decreases the velocity profile $f^{\prime}$;

- $\quad \gamma, N b$, Pr reduce the temperature field $\theta$;

- The wall thickness of the motile microorganism $\chi$ is a declining function of $P e, L b, \sigma$;

- The density of the motile microorganism $\chi$ is an enhancing function of $M, N t$;

- $\quad \mathrm{Nb}$, Le regulate the concentration profile;

- The concentration field increases with the rise of $\gamma, M, N t$ and decreases with the increase of $\mathrm{Nb}$, Le.

Nanoparticle comprised microorganisms find notable applications in tissue engineering, food processing, and biopolymer processing. In future, we want to extend the concept by considering induced magnetic fields and try to figure out its error analysis.

Author Contributions: First author developed the conceptualization, theory, computations and writing the original draft. Second author developed visualization, investigation, review and editing. Third and fourth author review, resources, software and funding acquisition. All authors discussed the results and contributed to the final manuscripts.

Funding: This research received no external funding.

Conflicts of Interest: The authors declare no conflict of interest.

\section{Nomenclature}

$a$

$B_{0}$

$C$

$C_{f}$

$C_{w}$

$C_{\infty}$

$c_{p}$

$D_{B}$

$D_{n}$

$D_{T}$

Ec

$L b$

Le

$l$

$M$

$\mathrm{Nb}$

$N t$

$N n_{x}$

$N u_{x}$

$n$
Radius of the cylinder

Magnetic field of strength

Concentration

Skin friction coefficient

Concentration at the surface

Concentration in the free stream

Specific heat at constant pressure

Brownian diffusion coefficient

Diffusivity of the microorganism

Thermophoresis coefficient

Eckert number

Bioconvection Lewis number

Lewis number

Characteristic length.

Magnetic field parameter

Brownian motion parameter

Thermophoresis parameter

Density number of the motile microorganism

Local Nusselt number

Density of the motile microorganism 


$\begin{array}{ll}n_{w} & \text { Constant motile microorganism } \\ P e & \text { Peclet number } \\ \operatorname{Pr} & \text { Prandtl number } \\ q_{n} & \text { Surface motile microorganism flux. } \\ q_{m} & \text { Surface mass flux } \\ q_{w} & \text { Surface heat flux } \\ \operatorname{Re} & \text { Reynolds number } \\ S h_{x} & \text { Local Sherwood number } \\ T & \text { Indicates the temperature } \\ T_{w} & \text { Constant temperature } \\ u & \text { Velocity parts along } x \text { directions } \\ U_{0} & \text { Constant } \\ U_{w} & \text { Axial route having velocity } \\ W c & \text { Constant maximum cell swimming speed } \\ v & \text { Velocity parts along } r \text { directions } \\ G r e e k \text { letters } & \\ \sigma & \text { Bioconvection parameter } \\ \sigma_{e} & \text { Electrical conductivity } \\ \alpha & \text { Thermal diffusivity } \\ \tau & \text { Effective heat capacitance } \\ v & \text { Kinematic viscosity } \\ \gamma & \text { Curvature parameter } \\ \tau_{w} & \text { Surface shear stress }\end{array}$

\section{References}

1. Schlichting, H.; Gersten, K.; Krause, E.; Oertel, H. Boundary-Layer Theory; McGraw-Hill: New York, NY, USA, 1960; Volume 7.

2. Sakiadis, B.C. Boundarylayer behavior on continuous solid surfaces: I. Boundarylayer equations for two dimensional and axi-symmetric flow. AIChE J. 1961, 7, 26-28. [CrossRef]

3. Sakiadis, B.C. Boundary-layer behavior on continuous solid surfaces: II. The boundary layer on a continuous flat surface. AIChE J. 1961, 7, 221-225. [CrossRef]

4. Crane, L.J. Flow past a stretching plate. Z. Angew. Math. Phys. 1970, 21, 645-647. [CrossRef]

5. Dutta, B.; Roy, P.; Gupta, A. Temperature field in flow over a stretching sheet with uniform heat flux. Int. Commun. Heat Mass Transf. 1985, 12, 89-94. [CrossRef]

6. Chen, C.-K.; Char, M.-I. Heat transfer of a continuous, stretching surface with suction or blowing. J. Math. Anal. Appl. 1988, 135, 568-580. [CrossRef]

7. Gupta, P.S.; Gupta, A.S. Heat and mass transfer on a stretching sheet with suction or blowing. Can. J. Chem. Eng. 1977, 55, 744-746. [CrossRef]

8. Hayat, T.; Javed, T.; Abbas, Z. Slip flow and heat transfer of a second grade fluid past a stretching sheet through a porous space. Int. J. Heat Mass Transf. 2008, 51, 4528-4534. [CrossRef]

9. Hayat, T.; Abbas, Z.; Sajid, M. Series solution for the upper-convected Maxwell fluid over a porous stretching plate. Phys. Lett. A 2006, 358, 396-403. [CrossRef]

10. Xu, H.; Liao, S.J. Analytic solutions of magnetohydrodynamic flows of non- Newtonian fluids caused by an impulsively stretching plate. J. Non-Newton Fluid Mech. 2005, 159, 46-55. [CrossRef]

11. Cortell, R. Flow and heat transfer of a fluid through a porous medium over a stretching surface with internal heat generation/absorption and suction/blowing. Fluid Dyn. Res. 2005, 37, 231-245. [CrossRef]

12. Cortell, R. Effects of viscous dissipation and work done by deformation on the MHD flow and heat transfer of a viscoelastic fluid over a stretching sheet. Phys. Lett. A 2006, 357, 298-305. [CrossRef]

13. Datta, P.; Anilkumar, D.; Roy, S.; Mahanti, N.C. Effect of non-uniform slot injection (suction) on a forced flow over a slender cylinder. Int. J. Heat Mass Transf. 2006, 49, 2366-2371. [CrossRef]

14. Elbarbary, E.M.E.; Elgazery, N.S. Flow and heat transfer of a micropolar fluid in an axi-symmetric stagnation flow on a cylinder with variable properties and suction (numerical study). Acta Mech. 2005, 76, 213-229. [CrossRef] 
15. Wang, C.Y. Fluid flow due to a stretching cylinder. Phys. Fluids 1988, 31, 466. [CrossRef]

16. Ishak, A.; Nazar, R.; Pop, I. Magnetohydrodynamic (MHD) flow and heat transfer due to a stretching cylinder. Energy Convers. Manag. 2008, 49, 3265-3269. [CrossRef]

17. Ishak, A.; Nazar, R.; Pop, I. Uniform suction/blowing effect on flow and heat transfer due to a stretching cylinder. Appl. Math. Model. 2008, 32, 2059-2066. [CrossRef]

18. Butt, A.S.; Ali, A.; Mehmood, A. Numerical investigation of magnetic field effects on entropy generation in viscous flow over a stretching cylinder embedded in a porous medium. Energy 2016, 99, 237-249. [CrossRef]

19. Choi, S.U.S.; Zhang, Z.G.; Yu, W.; Lockwood, F.E.; Grulke, E.A. Anomalous thermal conductivity enhancement in nanotube suspensions. Appl. Phys. Lett. 2001, 79, 2252-2254. [CrossRef]

20. Masuda, H.; Ebata, A.; Teramae, K. Alteration of thermal conductivity and viscosity of liquid by dispersing ultra-fine particles. Dispersion of $\mathrm{Al}_{2} \mathrm{O}_{3}, \mathrm{SiO}_{2}$ and $\mathrm{TiO}_{2}$ ultra-fine particles. Jpn. Soc. Thermophys. Prop. 1993, 7, 227-233. [CrossRef]

21. Platt, J.R. "Bioconvection Patterns" in Cultures of Free-Swimming organisms. Science 1961, 133, $1766-1767$. [CrossRef]

22. Kuznetsov, A. Bio-thermal convection induced by two different species of microorganisms. Int. Commun. Heat Mass Transf. 2011, 38, 548-553. [CrossRef]

23. Kuznetsov, A. The onset of thermo-bioconvection in a shallow fluid saturated porous layer heated from below in a suspension of oxytactic microorganisms. Eur. J. Mech. B Fluids 2006, 25, 223-233. [CrossRef]

24. Nield, D.A.; Kuznetsov, A.V. The onset of bio-thermal convection in a suspension of gyrotactic microorganisms in a fluid layer: Oscillatory convection. Int. J. Therm. Sci. 2006, 45, 990-997. [CrossRef]

25. Avramenko, A.A.; Kuznetsov, A.V. Stability of a suspension of gyrotactic microorganisms in superimposed fluid and porous layers. Int. Commun. Heat Mass Transf. 2004, 31, 1057-1066. [CrossRef]

26. Alloui, Z.; Nguyen, T.H.; Bilgen, E. Numerical investigation of thermo-bioconvection in a suspension of gravitactic microorganisms. Int. Commun. Heat Mass Transf. 2007, 50, 1435-1441. [CrossRef]

27. Mahdy, A. Gyrotactic Microorganisms Mixed Convection Nanofluid Flow along an Isothermal Vertical Wedge in Porous Media. Int. Sch. Sci. Res. Innov. 2017, 11, 829-839.

28. Tham, L.; Nazar, R.; Pop, I. Steady Mixed Convection Flow on a Horizontal Circular Cylinder Embedded in a Porous Medium Filled by a Nanofluid Containing Gyrotactic Micro-Organisms. J. Heat Transf. 2013, 135, 102601. [CrossRef]

29. Kuznetsov, A. The onset of nanofluid bioconvection in a suspension containing both nanoparticles and gyrotactic microorganisms. Int. Commun. Heat Mass Transf. 2010, 37, 1421-1425. [CrossRef]

30. Buongiorno, J. Convective transport in nanofluids. J. Heat Transf. 2006, 128, 240-250. [CrossRef]

31. Dinarvand, S.; Hosseini, R.; Pop, I. Unsteady convective heat and mass transfer of a nanofluid in Howarth's stagnation point by Buongiorno's model. Int. J. Numer. Methods Heat Fluid Flow 2015, 25, 1176-1197. [CrossRef]

32. Dinarvand, S.; Abbassi, A.; Hosseini, R.; Pop, I. Homotopy analysis method for mixed convective boundary layer flow of a nanofluid over a vertical circular cylinder. Therm. Sci. 2015, 19, 549-561. [CrossRef]

33. Babu, M.J.; Sandeep, N. Effect of nonlinear thermal radiation on non-aligned bio-convective stagnation point flow of a magnetic-nanofluid over a stretching sheet. Alex. Eng. J. 2016, 55, 1931-1939. [CrossRef]

34. Khan, W.; Pop, I.; Khan, W. Boundary-layer flow of a nanofluid past a stretching sheet. Int. J. Heat Mass Transf. 2010, 53, 2477-2483. [CrossRef]

35. Wang, C.Y. Free Convection on a Vertical Stretching Surface. ZAMM 1989, 69, 418-420. [CrossRef]

36. Mukhopadhyay, S. MHD boundary layerslip flow along a stretching cylinder. Ain Shams Eng. J. 2013, 4, 317-324. [CrossRef]

37. Motsa, S.S. A new spectral relaxation method for similarity variable nonlinear boundary layer flow systems. Chem. Eng. Commun. 2014, 201, 241-256. [CrossRef]

38. Motsa, S.S.; Makukula, Z.G. On spectral relaxation method approach for steady von Kármán flow of a Reiner-Rivlin fluid with Joule heating, viscous dissipation and suction/injection. Cent. Eur. J. Phys. 2013, 11, 363-374. [CrossRef]

39. Shateyi, S. A new numerical approach to MHD flow of a maxwelfluid past a vertical stretching sheet in the presence of thermophoresis and chemical reaction. Bound. Value Probl. 2013, 2013, 196. [CrossRef]

40. Shateyi, S.; Makinde, O.D. Hydromagnetic stagnation-point flow towards a radially stretching convectively heated disk. Math. Probl. Eng. 2013, 2013, 616947. [CrossRef] 
41. Shateyi, S.; Marewo, G.T. A new numerical approach of MHD flow with heat and mass transfer for the UCM fluid over a stretching surface in the presence of thermal radiation. Math. Probl. Eng. 2013, 2013, 670205. [CrossRef]

42. Motsa, S.S.; Dlamini, P.G.; Khumalo, M. Solving hyperchaotic systems using the spectral relaxation method. Abstr. Appl. Anal. 2012, 2012, 203461. [CrossRef]

43. Motsa, S.S.; Dlamini, P.; Khumalo, M. A new multistage spectral relaxation method for solving chaotic initial value systems. Nonlinear Dyn. 2013, 72, 265-283. [CrossRef]

44. SaberiNik, H.; Rebelo, P. Multistage spectral relaxation method for solving the hyperchaotic complex systems. Sci. World J. 2014, 2014, 943293.

45. Motsa, S.S.; Dlamini, P.G.; Khumalo, M. Spectral relaxation method and spectral quasilinearization method for solving unsteady boundary layer flow problems. Adv. Math. Phys. 2014, 2014, 341964. [CrossRef]

46. Awad, F.G.; Motsa, S.; Khumalo, M. Heat and mass transfer in unsteady rotating fluid flow with binary chemical reaction and activation energy. PLOS ONE 2014, 9, e107622. [CrossRef] [PubMed]

47. Haroun, N.A.; Sibanda, P.; Mondal, S.; Motsa, S.S. On unsteady MHD mixed convection in a nanofluid due to a stretching/shrinking surface with suction/injection using the spectral relaxation method. Bound. Value Probl. 2015, 24, 1-17. [CrossRef]

48. Elbashbeshy, E.; Emam, T.G.; El-Azab, M.S.; Abdelgaber, K.M. Slip effects on flow, heat, and mass transfer of nanofluid over stretching horizontal cylinder in the presence of suction/injection. Therm. Sci. 2014, 135. [CrossRef]

(C) 2019 by the authors. Licensee MDPI, Basel, Switzerland. This article is an open access article distributed under the terms and conditions of the Creative Commons Attribution (CC BY) license (http://creativecommons.org/licenses/by/4.0/). 